\title{
molecules
}

ISSN 1420-3049

www.mdpi.com/journal/molecules

Article

\section{Discovery of Hybrid Dual $N$-Acylhydrazone and Diaryl Urea Derivatives as Potent Antitumor Agents: Design, Synthesis and Cytotoxicity Evaluation}

\section{Xin Zhai, Qiang Huang, Nan Jiang, Di Wu, Hongyu Zhou and Ping Gong *}

Key Laboratory of Structure-Based Drug Design \& Discovery, Ministry of Education, School of Pharmaceutical Engineering, Shenyang Pharmaceutical University, Shenyang 110016, Liaoning, China

* Author to whom correspondence should be addressed; E-Mail: gongpinggp@126.com;

Tel./Fax: +86-24-2398-6429.

Received: 7 January 2013; in revised form: 19 January 2013 / Accepted: 25 January 2013 /

Published: 4 March 2013

\begin{abstract}
Based on the hybrid pharmacophore design concept, a novel series of dual diaryl urea and $\mathrm{N}$-acylhydrazone derivatives were synthesized and evaluated for their in vitro cytotoxicity by the standard MTT assay. The pharmacological results indicated that most compounds exhibited moderate to excellent activity. Moreover, compound 2g showed the most potent cytotoxicity against HL-60, A549 and MDA-MB-231 cell lines, with $\mathrm{IC}_{50}$ values of $0.22,0.34$ and $0.41 \mu \mathrm{M}$, respectively, which was 3.8 to 22.5 times more active than the reference compounds sorafenib and PAC-1. The promising compound $\mathbf{2 g}$ thus emerges as a lead for further structural modifications.
\end{abstract}

Keywords: diaryl ureas; $N$-acylhydrazone; cytotoxicity

\section{Introduction}

Design of single chemical compounds that simultaneously modulate multiple biological targets in a specific manner is the current focus of new drug development and is becoming more popular. An effective approach is to take existing individual compounds, each known to have pharmacological structural features and high selectivity against the particular targets of interest, and combine them into a single molecule. Sorafenib (Figure 1), a diaryl urea analogue [1], is a small molecular inhibitor of several tyrosine protein kinases (VEGFR, PDGFR and B-Raf) [2,3] and unique in targeting the 
Raf/Mek/Erk pathway (MAPK pathway) [4], was approved by FDA for the treatment of advanced renal cell carcinoma and advanced hepatocellular carcinoma [5,6]. PAC-1 (Figure 1), the first preferential small molecule procaspase-3 activating compound with $N$-acylhydrazone pharmacophore, is promising as a new anti-tumor drug that can directly influence the apoptotic machinery or suicide of cells and has shown good results in mouse models [7-9].

Figure 1. Structures of sorafenib, PAC-1 and target compounds.<smiles>CNC(=O)c1cc(Oc2ccc(NC(=O)Nc3ccc(Cl)c(C(F)(F)F)c3)cc2)ccn1</smiles><smiles>C=CCc1cccc(/C=N/NC(=O)CN2CCN(Cc3ccccc3)CC2)c1O</smiles>

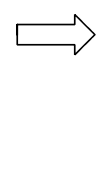

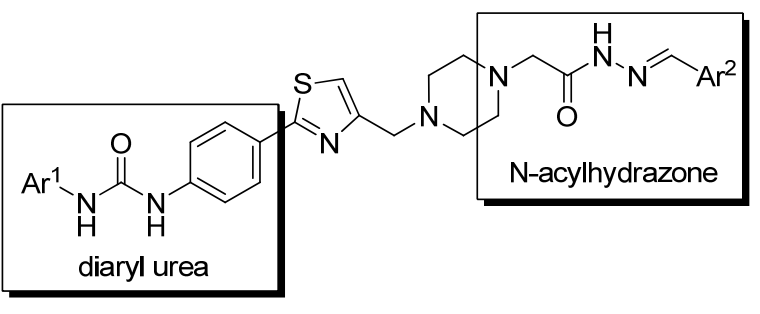

1a-g, 2a-k, 3a-e, 4a-c

In an attempt to discover new antitumor agents with multiple molecular mechanisms, we combined the diaryl urea moiety from sorafenib and $N$-acylhydrazone based in a hybrid pharmacophore design. The use of thiazolyl and amido moieties as linkers has been reported in our preliminary study [10], and the thiazolyl ring is retained in this paper for its better antitumor potency. Thus a series of diaryl urea derivatives bearing an $\mathrm{N}$-acylhydrazone moiety (Figure 1) were designed and synthesized. Various substituted ureido-linked phenyl $\left(\mathrm{Ar}^{1}\right)$ and hydrazone-linked phenyl $\left(\mathrm{Ar}^{2}\right)$ groups were introduced to explore the influence of electronic and steric effects on the anticancer activity. 2-Hydroxyl substitution was retained for the $\mathrm{Ar}^{2}$ ring for the reason that only with the hydroxyl group on $\mathrm{Ar}^{2}$ did the PAC-1 derivatives display antitumor activity in vitro [11]. 4-and 5-Benzyloxyl groups were introduced to $\mathrm{Ar}^{2}$, respectively, to investigate the effect of the extension of the hydrophobic region. Furthermore, $\mathrm{Ar}^{2}$ was replaced with a substituted chromenonyl or imidazolindionyl groups, which are often associated with a variety of biological activity, to note the effect of each discreet change on the biological activity of the resulting compounds.

\section{Results and Discussion}

\subsection{Chemistry}

The synthesis of target compounds $1 \mathbf{a}-\mathbf{g}, \mathbf{2 a}-\mathbf{k}, \mathbf{3 a}-\mathbf{e}$ and $4 \mathbf{a}-\mathbf{c}$ is described in Scheme 1. Commercially available 4-aminobenzonitrile reacted with triphosgene in dioxane at $80{ }^{\circ} \mathrm{C}$ for $24 \mathrm{~h}$ to give 4-isocyanatobenzonitrile (5) as a colorless oil. Compound $\mathbf{5}$ was treated with various substituted anilines to obtain diaryl ureas $\mathbf{6 a}-\mathbf{0}$ [12], whose cyano group was reduced to a thioamide moiety using magnesium chloride and sodium hydrogensulfide in $N, N$-dimethylformamide to afford the corresponding derivatives $7 \mathbf{a}-\mathbf{o}$. Cyclization of $\mathbf{7 a - 0}$ with 1,3-dichloroacetone in tetrahydrofuran at 50 ${ }^{\circ} \mathrm{C}$ readily afforded thiozoles $\mathbf{8 a}-\mathbf{0}$, which reacted with piperazine in ethanol by nucleophilic substitution to give 9a-o. Consequently, treatment of 9a-o with ethyl chloroacetate in ethanol in the presence of potassium carbonate and sodium iodide afforded esters $\mathbf{1 0 a}-\mathbf{0}$, which were turned into 
acylhydrazines 11a-o via hydrazinolysis in $80 \%$ hydrazine hydrate for $48 \mathrm{~h}$. Finally, target compounds 1a-g, 2a-k, 3a-e and 4a-c were prepared via condensation of 11a-o with substituted benzaldehydes, various aromatic aldehydes $(\mathbf{1 2 a}-\mathbf{d}, \mathbf{1 3 a}-\mathbf{c}$ or $\mathbf{1 5})$ as well as imidazolindiones $\mathbf{1 9 a}-\mathbf{b}$, respectively, and isolated as the corresponding dihydrochlorides [7].

Scheme 1. Synthesis of target compounds $1 \mathbf{a}-\mathbf{g}, \mathbf{2 a}-\mathbf{k}, 3 \mathbf{3}-\mathbf{e}$ and $4 \mathbf{a}-\mathbf{c}$.

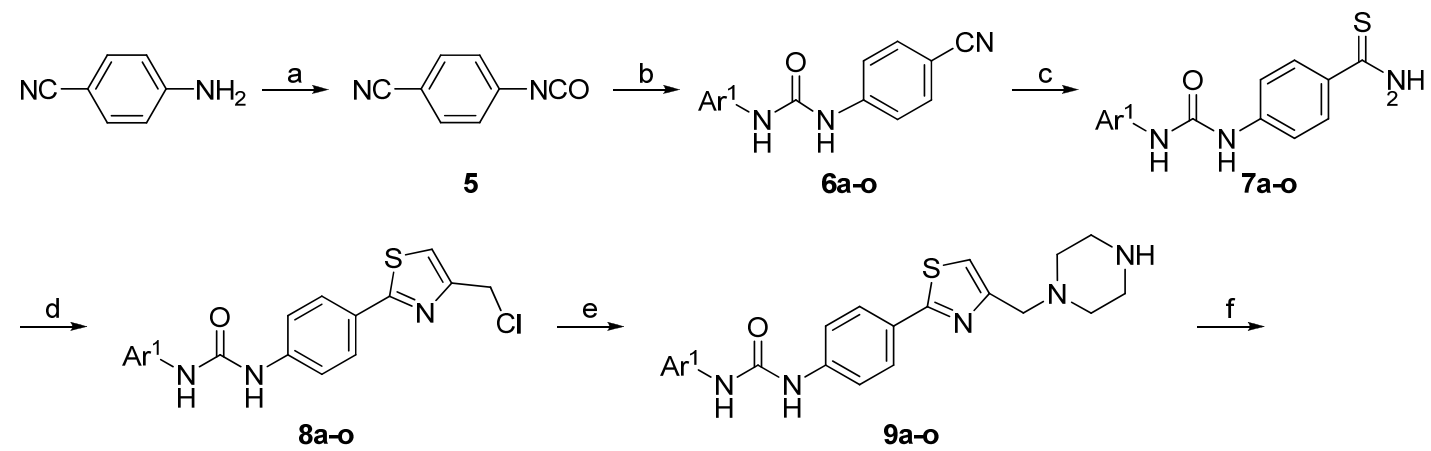<smiles>CCCNC(=O)Nc1ccc(-c2nc(CN3CCN(CC(=O)OCC)CC3)cs2)cc1</smiles>

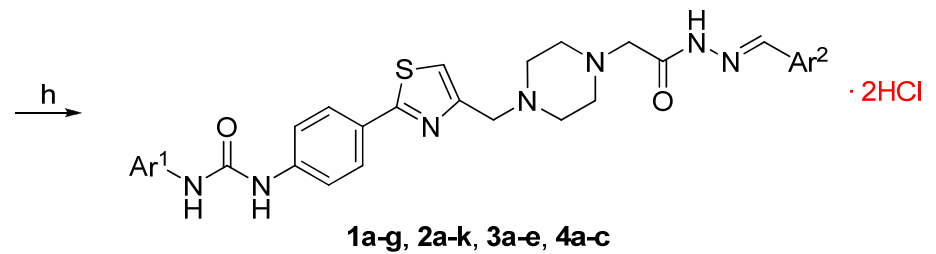
6a-11a: $\mathrm{Ar}^{1}=3-\mathrm{F}-\mathrm{C}_{6} \mathrm{H}_{4}$
6b-11b: $\mathrm{Ar}^{1}=3,5-\mathrm{Cl}-\mathrm{C}_{6} \mathrm{H}_{3}$
6c-11c: $\mathrm{Ar}^{1}=3-\mathrm{Cl}-4-\mathrm{F}-\mathrm{C}_{6} \mathrm{H}_{3}$
6f-11f: $\mathrm{Ar}^{1}=3-\mathrm{Cl}-\mathrm{C}_{6} \mathrm{H}_{4}$ 6g-11g: $\mathrm{Ar}^{1}=3,4-\mathrm{Cl}-\mathrm{C}_{6} \mathrm{H}_{3}$ 6h-11h: $\mathrm{Ar}^{1}=3-\mathrm{OCF}_{3}-\mathrm{C}_{6} \mathrm{H}_{4}$ 6d-11d: $\mathrm{Ar}^{1}=4-\mathrm{CF}_{3}-\mathrm{C}_{6} \mathrm{H}_{4}$ 6e-11e: $\mathrm{Ar}^{1}=3-\mathrm{CF}_{3}-\mathrm{C}_{6} \mathrm{H}_{4}$ 6i-11i: $\mathrm{Ar}^{1}=\mathrm{C}_{6} \mathrm{H}_{5}$ 6j-11j: $\mathrm{Ar}^{1}=2-\mathrm{CF}_{3}-\mathrm{C}_{6} \mathrm{H}_{4}$
6k-11k: $\mathrm{Ar}^{1}=4-\mathrm{Cl}-3-\mathrm{CF}_{3}-\mathrm{C}_{6} \mathrm{H}_{3}$
6I-11I: $\mathrm{Ar}^{1}=3-\mathrm{OCH}_{3}-\mathrm{C}_{6} \mathrm{H}_{4}$
6m-11m: $\mathrm{Ar}^{1}=3,5-\mathrm{CF}_{3}-\mathrm{C}_{6} \mathrm{H}_{3}$
6n-11n: $\mathrm{Ar}^{1}=3,4-\mathrm{CH}_{3}-\mathrm{C}_{6} \mathrm{H}_{3}$
6o-11o: $\mathrm{Ar}^{1}=3-\mathrm{Cl}_{3}-\mathrm{CF}_{3}-\mathrm{C}_{6} \mathrm{H}_{3}$

Reactions and conditions: (a) triphosgene, dioxane, $80{ }^{\circ} \mathrm{C}, 24 \mathrm{~h}$; (b) $\mathrm{Ar}^{1} \mathrm{NH}_{2}$, THF, r.t.; (c) $\mathrm{MgCl}_{2}, \mathrm{NaSH}$, DMF, overnight; (d) $\mathrm{ClCH}_{2} \mathrm{COCH}_{2} \mathrm{Cl}$, THF, $50{ }^{\circ} \mathrm{C}, 7 \mathrm{~h}$; (e) piperazine, EtOH, r.t., 2 h; (f) $\mathrm{ClCH}_{2} \mathrm{COOEt}$, $\mathrm{K}_{2} \mathrm{CO}_{3}$, NaI, EtOH, $50{ }^{\circ} \mathrm{C}, 2 \mathrm{~h}$; (g) $80 \% \mathrm{NH}_{2} \mathrm{NH}_{2} \cdot \mathrm{H}_{2} \mathrm{O}$, EtOH, $50{ }^{\circ} \mathrm{C}$, $48 \mathrm{~h}$; (h) i. ArCHO, 12a-d, 13a-c or 15, EtOH, then $\mathrm{HCl}-\mathrm{EtOH}$; ii. 19a-b, $\mathrm{HCl}, \mathrm{EtOH}$, then $\mathrm{HCl}-\mathrm{EtOH}$.

As shown in Scheme 2, aryloxybenzaldehydes 12a-d and 13a-c were prepared from 2,5-(or 2,4-) dihydroxybenzaldehyde via regioselective $O$-alkylation reactions with benzyl chlorides in acetonitrile in the presence of sodium hydrogencarbonate and potassium iodide. Cyclization of $m$-dihydroxybenzene with ethyl acetoacetate in sulfuric acid at $10{ }^{\circ} \mathrm{C}$, followed by formylation with urotropine in glacial acetic acid and sulfuric acid in sequence, namely a Duff reaction, provided chromenealdehyde $\mathbf{1 5}$ as a white solid [13]. Imidazolindiones 19a-b were synthesized from substituted anilines, which were turned into phenyl isocyanates $\mathbf{1 6 a}-\mathbf{b}$ in a similar manner as described for compound $\mathbf{5}$. Subsequent treatment of $\mathbf{1 6} \mathbf{a}-\mathbf{b}$ with glycine methyl ester hydrochloride in the presence of triethylamine in dichloromethane gave ureas $17 \mathbf{a}-\mathbf{b}$. Cyclization of $17 \mathbf{a}-\mathbf{b}$ in the presence of concentrated hydrochloride gave rise to $18 \mathbf{a}-\mathbf{b}$, which were further condensed with $N, N$-dimethylformamide dimethylacetal (DMF-DMA) to obtain 19a-b [14]. 
Scheme 2. Synthesis of intermediates $12 \mathbf{a}-\mathbf{d}, \mathbf{1 3 a}-\mathbf{c}, 15$ and $19 \mathbf{a}-\mathbf{b}$.

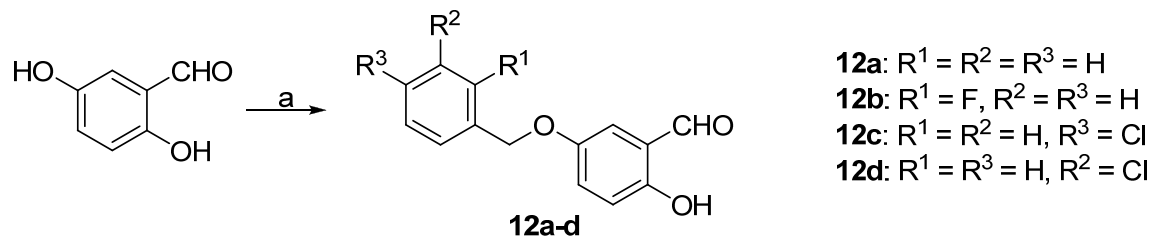

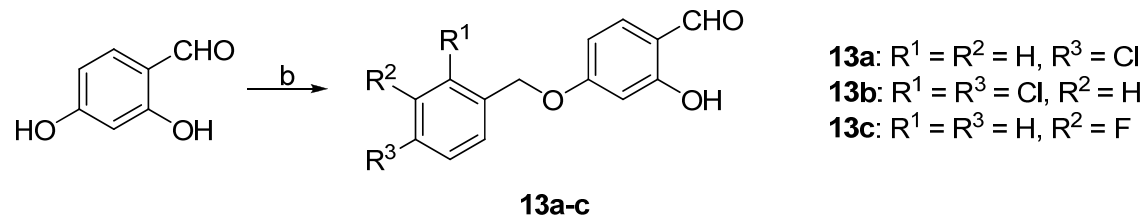<smiles>Oc1cccc(O)c1</smiles><smiles>Cc1cc(=O)oc2cc(O)ccc12</smiles><smiles>Cc1cc(=O)oc2cc(O)c(C=O)cc12</smiles><smiles>[R]c1ccc(NC(=O)NC(=O)Nc2ccc([R])cc2)cc1</smiles><smiles>[R]c1ccc(N2C(=O)N/C(=C/N(C)C)C2=O)cc1</smiles>

Reactions and conditions: (a) $\mathrm{ArCH}_{2} \mathrm{Cl}, \mathrm{NaHCO}_{3}, \mathrm{CH}_{3} \mathrm{CN}$, r.f., 30 h; (b) $\mathrm{ArCH}_{2} \mathrm{Cl}, \mathrm{NaHCO}_{3}, \mathrm{CH}_{3} \mathrm{CN}$, r.f., 30 h; (c) ethyl acetoacetate, $\mathrm{H}_{2} \mathrm{SO}_{4}, 10^{\circ} \mathrm{C}, 4 \mathrm{~h}$; (d) urotropine, $\mathrm{HOAc}, 115^{\circ} \mathrm{C}, 4 \mathrm{~h}$; (e) triphosgene, dioxane, $80^{\circ} \mathrm{C}$, 24 h; (f) Glycine methyl ester hydrochloride, TEA, $\mathrm{CH}_{2} \mathrm{Cl}_{2}$, r.t., 1 h, $0^{\circ} \mathrm{C}, 4$ h, r.t. 1 h; (g) conc. $\mathrm{HCl}$, acetone, 4 h; (h) DMF-DMA, $\mathrm{CH}_{3} \mathrm{CN}$, r.f., 4 h.

\subsection{Biological Results and Discussion}

All target compounds $\mathbf{1 a}-\mathbf{g}, \mathbf{2 a}-\mathbf{k}, \mathbf{3 a}-\mathbf{e}$ and $\mathbf{4 a}-\mathbf{c}$ were evaluated for their cytotoxicity in vitro against the human leukemia cell line (HL-60), human lung adenocarcinoma epithelial cell line (A549) and human breast cancer cell line (MDA-MB-231) by the 3-(4,5-dimethylthiazol-2-yl)-2,5diphenyltetrazolium bromide (MTT) assay, taking sorafenib and PAC-1 as references. The results expressed as $\mathrm{IC}_{50}$ values are summarized in Table 1 . The $\mathrm{IC}_{50}$ values are the average of at least three independent experiments. As listed in Table 1, phenylhydrazones 1a-g and 2a-k, with the exception of 1c and 1d, exhibited moderate to excellent cytotoxicity towards the tested cell lines with $\mathrm{IC}_{50}$ values ranging from 0.22 to $6.0 \mu \mathrm{m}$. Generally, phenyl groups $\mathrm{Ar}^{2}$ substituted with chromenyl and imidazolidinyl moieties gave rise to two series of compounds $\mathbf{3 a}-\mathbf{e}$ and $\mathbf{4 a}-\mathbf{c}$ with a dramatic decrease or even a loss in antitumor potency, indicating that $\mathrm{Ar}^{2}$ was critical for the optimal activity and it is not tolerant ofr bulky and rigid heteroaromatic rings in this region. 
Table 1. Structures and cytotoxicity of compounds $1 \mathbf{a}-\mathbf{g}, \mathbf{2 a}-\mathbf{k}, \mathbf{3 a}-\mathbf{e}$ and $4 \mathbf{a}-\mathbf{c}$ against HL-60, A549 and MDA-MB-231 cell lines.

\begin{tabular}{|c|c|c|c|c|c|}
\hline \multirow{2}{*}{ Compd. } & \multirow{2}{*}{$\mathbf{A r}{ }^{1}$} & \multirow{2}{*}{$A \mathbf{r}^{2}$} & \multicolumn{3}{|c|}{$\mathrm{IC}_{50}(\mu \mathrm{mol} / \mathrm{L})$} \\
\hline & & & HL-60 & A549 & MDA-MB-231 \\
\hline $1 \mathrm{a}$ & & & ND & $0.64 \pm 0.12$ & $1.9 \pm 0.16$ \\
\hline $1 b$ & & & $0.56 \pm 0.04$ & $0.78 \pm 0.02$ & $0.48 \pm 0.02$ \\
\hline $1 \mathrm{c}$ & & & $13.0 \pm 0.37$ & $0.48 \pm 0.06$ & $0.26 \pm 0.01$ \\
\hline 1d & & & $8.8 \pm 0.31$ & $5.1 \pm 0.25$ & $8.5 \pm 0.44$ \\
\hline $1 \mathrm{e}$ & & & $0.82 \pm 0.08$ & $1.6 \pm 0.41$ & $0.92 \pm 0.24$ \\
\hline 1f & & & $0.63 \pm 0.17$ & $1.3 \pm 0.16$ & $0.82 \pm 0.05$ \\
\hline $1 \mathrm{~g}$ & & & $6.0 \pm 0.09$ & $0.50 \pm 0.04$ & $0.58 \pm 0.03$ \\
\hline $2 a$ & & & $0.55 \pm 0.09$ & $1.6 \pm 0.14$ & $0.73 \pm 0.06$ \\
\hline $2 b$ & & & $0.51 \pm 0.01$ & $1.2 \pm 0.05$ & $0.73 \pm 0.02$ \\
\hline $2 c$ & & & $2.6 \pm 0.11$ & $0.59 \pm 0.02$ & $0.71 \pm 0.01$ \\
\hline $2 d$ & & & $3.8 \pm 0.13$ & $1.7 \pm 0.12$ & $0.53 \pm 0.02$ \\
\hline $2 e$ & & & $2.3 \pm 0.11$ & $0.49 \pm 0.05$ & $0.35 \pm 0.02$ \\
\hline $2 f$ & & & $4.7 \pm 0.19$ & $2.8 \pm 0.21$ & $0.48 \pm 0.05$ \\
\hline $2 g$ & & & $0.22 \pm 0.01$ & $0.34 \pm 0.01$ & $0.41 \pm 0.3$ \\
\hline
\end{tabular}


Table 1. Cont.

\begin{tabular}{|c|c|c|c|c|c|}
\hline \multirow{2}{*}{ Compd. } & \multirow{2}{*}{$A \mathbf{r}^{1}$} & \multirow{2}{*}{$A \mathbf{r}^{2}$} & \multicolumn{3}{|c|}{$\mathrm{IC}_{50}(\mu \mathrm{mol} / \mathrm{L})$} \\
\hline & & & HL-60 & A549 & MDA-MB-231 \\
\hline $2 \mathrm{~h}$ & & & $0.50 \pm 0.004$ & $1.8 \pm 0.04$ & $0.90 \pm 0.006$ \\
\hline $2 \mathbf{i}$ & & & $0.38 \pm 0.01$ & $0.54 \pm 0.06$ & $0.44 \pm 0.04$ \\
\hline $2 j$ & & & $0.31 \pm 0.14$ & $0.96 \pm 0.20$ & $2.0 \pm 0.12$ \\
\hline $2 \mathbf{k}$ & & & $2.0 \pm 0.11$ & $2.3 \pm 0.08$ & $0.22 \pm 0.04$ \\
\hline $3 \mathbf{a}$ & & & $15.2 \pm 0.22$ & $17.0 \pm 0.52$ & $5.6 \pm 0.36$ \\
\hline $3 \mathbf{b}$ & & & $3.6 \pm 0.12$ & $>50$ & $3.8 \pm 0.28$ \\
\hline $3 \mathbf{c}$ & & & $3.3 \pm 0.25$ & $6.4 \pm 0.42$ & $3.6 \pm 0.28$ \\
\hline 3d & & & $4.0 \pm 0.33$ & $1.7 \pm 0.15$ & $1.8 \pm 0.07$ \\
\hline $3 \mathbf{e}$ & & & $4.5 \pm 0.13$ & $19.0 \pm 0.57$ & $8.9 \pm 0.41$ \\
\hline $4 a$ & & & $12.0 \pm 0.32$ & $37.2 \pm 0.46$ & $7.0 \pm 0.18$ \\
\hline $4 b$ & & & $25.5 \pm 0.29$ & $3.4 \pm 0.10$ & $13.3 \pm 0.32$ \\
\hline $4 c$ & & & $>50$ & $7.8 \pm 0.20$ & $13.1 \pm 0.37$ \\
\hline Sorafenib & & & ND & $1.3 \pm 0.06$ & $2.7 \pm 0.11$ \\
\hline PAC-1 & & & $4.5 \pm 0.03$ & $2.8 \pm 0.10$ & $2.0 \pm 0.05$ \\
\hline
\end{tabular}

ND: not determined. 
Compounds with alkyl groups on the phenyl ring $\mathrm{Ar}^{2}$ (compounds 1a-g) exhibited moderate activity, however, the introduction of 4- or 5-benzyloxyl groups to the phenyl ring $\mathrm{Ar}^{2}$ resulted in a remarkable increase in the activity $(\mathbf{2 a}-\mathbf{c}, \mathbf{2 e}, \mathbf{2 g}-\mathbf{k})$. Moreover, compounds substituted with a 5-(2-fluorobenzyloxy) group ( $\mathbf{2}$ and $\mathbf{2} \mathbf{j}$ ) exhibited the most potent cytotoxicity, especially compound $2 \mathrm{~g}$ which displayed prominent activity with $\mathrm{IC}_{50}$ values of $0.22,0.34$ and $0.41 \mu \mathrm{M}$, respectively, which were 3.8- to 20.5-fold higher than those of sorafenib and PAC-1.

As for substituents on the ureido-linked phenyl $\mathrm{Ar}^{1}$, the introduction of electron-withdrawing substituents on $\mathrm{Ar}^{1}$ was beneficial to the improvement of cytotoxicity, and the trifluoromethoxyl group produced the best potency. A case in point is that compound $\mathbf{2 g}$ with a trifluoromethoxyl group at the meta-position of $\mathrm{Ar}^{1}$ showed more potent cytotoxicity against all the three tested cell lines than compound $\mathbf{2 h}$ with no substituent. Similarly, $\mathbf{3 b}$ and $\mathbf{3 d}$ with 3-trifluoromethyl and 3,5-di-trifluoromethyl groups on $\mathrm{Ar}^{1}$, respectively, displayed potency against two or three cell lines than 3a with no substituent, while 3e with an electron-donating 3,4-dimethyl group showed a decline in antitumor activity against the A549 and MDA-MB-231 cell lines.

\section{Experimental}

\subsection{Chemistry}

Melting points were obtained on a Büchi Melting Point B-540 apparatus (Büchi Labortechnik, Flawil, Switzerland) and were uncorrected. Mass spectra (MS) was taken in ESI mode on Agilent 1100 LC-MS (Agilent, Palo Alto, CA, USA). Proton $\left({ }^{1} \mathrm{H}\right)$ nuclear magnetic resonance spectroscopy was performed using a Bruker ARX-300 300 MHz spectrometers (Bruker Bioscience, Billerica, MA, USA) with TMS as an internal standard. Unless otherwise noted, all the materials were obtained from commercial available sources and were used without further purification.

\subsection{4-Isocyanatobenzonitrile (5)}

4-Aminobezonitrile (100 g, $0.847 \mathrm{~mol})$ was treated with excess hydrogen chloride-ethanol and the resulting solution was evaporated to dryness. The hydrochloride salt obtained was then dissolved in dioxane $(200 \mathrm{~mL})$ and added dropwise to a solution of triphosgene $(125 \mathrm{~g}, 0.423 \mathrm{~mol})$ in dioxane $(200 \mathrm{~mL})$. The reaction mixture was heated to $80{ }^{\circ} \mathrm{C}$ and stirred for $24 \mathrm{~h}$. The resulting mixture was concentrated in vacuo and distilled under reduced pressure to give 5 (103 g, 84.4\%) as colorless oil. B.p.: $130-131{ }^{\circ} \mathrm{C}(15 \mathrm{mmHg})$.

\subsection{General Procedure for Preparation of 1-(4-Cyanophenyl)-3-substituted Phenylureas 6a-0}

To a solution of $5(16 \mathrm{~g}, 0.111 \mathrm{~mol})$ in tetrahydrofuran $(100 \mathrm{~mL})$ was slowly added the corresponding substituted aniline $(0.111 \mathrm{~mol})$. The resulted mixture was stirred at room temperature and the reaction was monitored by TLC. The reaction mixture was concentrated in vacuo, and the precipitated product was filtered and dried to obtain $\mathbf{6 a - 0}$.

1-(4-Cyanophenyl)-3-(3-fluorophenyl)urea (6a): Yield: 87.2\%; ESI-MS m/z: $256.1[\mathrm{M}+\mathrm{H}]^{+}$. 1-(4Cyanophenyl)-3-(3,5-dichlorophenyl)urea (6b): Yield: 78.5\%; ESI-MS m/z: $306.0[\mathrm{M}+\mathrm{H}]^{+}$. 1-(4- 
Cyanophenyl-3-(3-chloro-4-fluorophenyl)urea (6c): Yield: 84.7\%; ESI-MS m/z: 290.0 [M+H] ${ }^{+}$. 1-(4Cyanophenyl)-3-(4-(trifluoromethyl)phenyl)urea (6d): Yield: 80.5\%; ESI-MS m/z: $306.1[\mathrm{M}+\mathrm{H}]^{+}$. 1-(4-Cyanophenyl)-3-(3-(trifluoromethyl)phenyl)urea (6e): Yield: 80.0\%; ESI-MS m/z: $306.1[\mathrm{M}+\mathrm{H}]^{+}$. 1-(4-Cyanophenyl)-3-(3-chlorophenyl)urea (6f): Yield: 88.5\%; ESI-MS m/z: $272.0[\mathrm{M}+\mathrm{H}]^{+}$. 1-(4Cyanophenyl)-3-(3,4-dichlorophenyl)urea (6g): Yield: 81.9\%; ESI-MS m/z: $306.0[\mathrm{M}+\mathrm{H}]^{+}$. 1-(4Cyanophenyl)-3-(3-(trifluoromethoxy)phenyl)urea (6h): Yield: 84.8\%; ESI-MS m/z: $322.1[\mathrm{M}+\mathrm{H}]^{+}$. 1-(4-Cyanophenyl)-3-phenylurea (6i): Yield: 79.8\%; ESI-MS m/z: $238.1[\mathrm{M}+\mathrm{H}]^{+} .1$-(4-Cyanophenyl)3-(2-(trifluoromethyl)phenyl)urea (6j): Yield: 83.4\%; ESI-MS m/z: $306.1[\mathrm{M}+\mathrm{H}]^{+} .1$ 1-(4-Cyanophenyl)3-(4-chloro-3-(trifluoromethyl)phenyl)urea (6k): Yield: 80.2\%; ESI-MS $m / z: 340.0[\mathrm{M}+\mathrm{H}]^{+}$. 1-(4Cyanophenyl)-3-(3-methoxyphenyl)urea (61): Yield: 83.0\%; ESI-MS m/z: $268.1[\mathrm{M}+\mathrm{H}]^{+}$. -(4Cyanophenyl)-3-(3,5-di(trifluoromethyl)phenyl)urea (6m): Yield: 82.8\%; ESI-MS m/z: 373.4 [M+H] . 1-(4-Cyanophenyl)-3-(3,4-dimethylphenyl)urea (6n): Yield: 82.4\%; ESI-MS m/z: $266.1[\mathrm{M}+\mathrm{H}]^{+}$. 1-(4Cyanophenyl)-3-(3-chloro-4-(trifluoromethyl)phenyl)urea (6o): Yield: 83.5\%; ESI-MS m/z: 340.0 [M+H] .

\subsection{General Procedure for Preparation of 4-(3-Substituted Phenylureido)benzothioamides 7a-0}

To a solution of benzonitrile $\mathbf{6 a - 0}(0.086 \mathrm{~mol})$ in $N, N$-dimethylformamide $(250 \mathrm{~mL})$ was added magnesium chloride (22 g, $0.109 \mathrm{~mol}$ ) and sodium hydrogensulfide (12.2 g, $0.218 \mathrm{~mol})$. The reaction mixture was stirred at room temperature overnight and then was added to $1 \mathrm{~L}$ water, acidified to $\mathrm{pH} 4$ with dilute hydrochloric acid and filtered. The collected solid was washed with water until the filtrate became neutral and dried to obtain $7 \mathbf{a}-\mathbf{0}$.

4-(3-(3-Fuorophenyl)ureido)benzothioamide (7a): Yield: 58.7\%; ESI-MS m/z: $290.1[\mathrm{M}+\mathrm{H}]^{+}$. 4-(3(3,5-Dichlorophenyl)ureido)benzothioamide (7b): Yield: 58.8\%; ESI-MS m/z: $340.0[\mathrm{M}+\mathrm{H}]^{+} .4-(3-(3-$ Chloro-4-fluorophenyl)ureido)benzothioamide (7c): Yield: 62.5\%; ESI-MS m/z:324.0 [M+H] $]^{+}$4-(3(4-(Trifluoromethyl)phenyl)ureido)benzothioamide (7d): Yield: 60.7\%; ESI-MS m/z: $340.1[\mathrm{M}+\mathrm{H}]^{+} .4-$ (3-(3-(Trifluoromethyl)phenyl)ureido)benzothioamide (7e): Yield: 66.4\%; ESI-MS m/z: $340.1[\mathrm{M}+\mathrm{H}]^{+}$. 4-(3-(3-Chlorophenyl)ureido)benzothioamide (7f): Yield: 59.4\%; ESI-MS m/z: $306.0[\mathrm{M}+\mathrm{H}]^{+}$. 4-(3(3,4-Dichlorophenyl)ureido)benzothioamide (7g): Yield: 67.0\%; ESI-MS m/z: $340.0[\mathrm{M}+\mathrm{H}]^{+} .4-(3-(3-$ (Trifluoromethoxy)phenyl)ureido)benzothioamide (7h): Yield: 59.2\%; ESI-MS m/z: $356.1[\mathrm{M}+\mathrm{H}]^{+} .4-$ (3-Phenylureido)benzothioamide (7i): Yield: 66.4\%; ESI-MS $m / z: 272.2 \quad[\mathrm{M}+\mathrm{H}]^{+}$. 4-(3-(2(Trifluoromethyl)phenyl)ureido)benzothioamide (7j): Yield: 67.2\%; ESI-MS m/z: $340.1[\mathrm{M}+\mathrm{H}]^{+}$. 4-(3(4-Chloro-3-(trifluoromethyl)phenyl)ureido)benzothioamide (7k): Yield: 61.1\%; ESI-MS m/z: 374.2 $[\mathrm{M}+\mathrm{H}]^{+}$. 4-(3-(3-Methoxyphenyl)ureido)benzothioamide (7l): Yield: 58.5\%; ESI-MS m/z:302.1 [M+H] . 4-(3-(3,5-Di(trifluoromethyl)phenyl)ureido)benzothioamide (7m): Yield: 62.7\%; ESI-MS m/z: 408.1 $[\mathrm{M}+\mathrm{H}]^{+}$. 4-(3-(3,4-Dimethylphenyl)ureido)benzothioamide (7n): Yield: 66.5\%; ESI-MS m/z:300.3 $[\mathrm{M}+\mathrm{H}]^{+}$. 4-(3-(3-Chloro-4-(trifluoromethyl)phenyl)ureido)benzothioamide (7o): Yield: 63.1\%; ESI-MS $m / z: 374.2[\mathrm{M}+\mathrm{H}]^{+}$. 
3.5. General Procedure for Preparation of 1-(4-(4-Chloromethylthiazol-2-yl)phenyl)-3-substituted Phenylureas 8a-0

Arylthioamides $7 \mathbf{a}-\mathbf{o}(0.083 \mathrm{~mol})$ were dissolved in tetrahydrofuran $(300 \mathrm{~mL})$ and heated to $50{ }^{\circ} \mathrm{C}$. To the stirred solution was added 1,3-dichloroacetone $(10.5 \mathrm{~g}, 0.083 \mathrm{~mol})$. The reaction mixture was stirred for $7 \mathrm{~h}$. The resulting mixture was evaporated in vacuo to remove most of the solvent, cooled and filtered off. The residue was suspended in $1 \mathrm{~L}$ water and the suspension was stirred and alkalinized to $\mathrm{pH} 8$ with saturated potassium carbonate solution. The precipitates was filtered, washed with water and dried to obtain $\mathbf{8 a}-\mathbf{0}$.

1-(4-(4-(Chloromethyl)thiazol-2-yl)phenyl)-3-(3-fluorophenyl)urea (8a): Yield: 50.3\%; ESI-MS m/z: $362.1[\mathrm{M}+\mathrm{H}]^{+}$. 1-(4-(4-(Chloromethyl)thiazol-2-yl)phenyl)-3-(3,5-dichlorophenyl)urea (8b): Yield: 58.1\%; ESI-MS m/z: $412.0[\mathrm{M}+\mathrm{H}]^{+}$. 1-(4-(4-(Chloromethyl)thiazol-2-yl)phenyl)-3-(3-chloro-4-fluorophenyl)urea (8c): Yield: 55.3\%; ESI-MS m/z: $396.0[\mathrm{M}+\mathrm{H}]^{+}$. 1-(4-(4-(Chloromethyl)thiazol-2-yl)phenyl)-3-(4(trifluoromethyl)phenyl)urea (8d): Yield: 53.5\%; ESI-MS m/z: $412.1[\mathrm{M}+\mathrm{H}]^{+}$. 1-(4-(4-(Chloromethyl) thiazol-2-yl)phenyl)-3-(3-(trifluoromethyl)phenyl)urea (8e): Yield: 50.9\%; ESI-MS m/z: $412.1[\mathrm{M}+\mathrm{H}]^{+}$. 1-(4-(4-(Chloromethyl)thiazol-2-yl)phenyl)-3-(3-chlorophenyl)urea (8f): Yield: 51.2\%; ESI-MS $\mathrm{m} / \mathrm{z}$ : $378.0[\mathrm{M}+\mathrm{H}]^{+}$. 1-(4-(4-(Chloromethyl)thiazol-2-yl)phenyl)-3-(3,4-dichlorophenyl)urea (8g): Yield: 57.5\%; ESI-MS m/z: $412.0[\mathrm{M}+\mathrm{H}]^{+}$. 1-(4-(4-(Chloromethyl)thiazol-2-yl)phenyl)-3-(3-(trifluoromethoxy) phenyl)urea (8h): Yield: 57.6\%; ESI-MS m/z: $428.1[\mathrm{M}+\mathrm{H}]^{+}$. 1-(4-(4-(Chloromethyl)thiazol-2-yl) phenyl)-3-phenylurea (8i): Yield: 54.3\%; ESI-MS m/z: $344.1[\mathrm{M}+\mathrm{H}]^{+}$. 1-(4-(4-(Chloromethyl)thiazol2-yl)phenyl)-3-(2-(trifluoromethyl)phenyl)urea (8j): Yield: 52.7\%; ESI-MS $m / z: 412.1[\mathrm{M}+\mathrm{H}]^{+} .1-(4-$ (4-(Chloromethyl)thiazol-2-yl)phenyl)-3-(4-chloro-3-(trifluoromethyl)phenyl)urea (8k): Yield: 55.6\%; ESI-MS $m / z: 446.0 \quad[\mathrm{M}+\mathrm{H}]^{+}$. 1-(4-(4-(Chloromethyl)thiazol-2-yl)phenyl)-3-(3-methoxyphenyl)urea (81): Yield: 57.1\%; ESI-MS m/z: $374.2[\mathrm{M}+\mathrm{H}]^{+}$. 1-(4-(4-(Chloromethyl)thiazol-2-yl)phenyl)-3-(3,5di(trifluoromethyl)phenyl)urea (8m): Yield: 52.3\%; ESI-MS m/z: $480.0[\mathrm{M}+\mathrm{H}]^{+}$. 1-(4-(4-(Chloromethyl) thiazol-2-yl)phenyl)-3-(3,4-dimethylphenyl)urea (8n): Yield: 50.4\%; ESI-MS m/z: 372.2 [M+H] $]^{+}$. 1-(4(4-(Chloromethyl)thiazol-2-yl)phenyl)-3-(3-chloro-4-(trifluoromethyl)phenyl)urea (8o): Yield: 54.2\%; ESI-MS $m / z: 446.0[\mathrm{M}+\mathrm{H}]^{+}$.

3.6. General Procedure for Preparation of 1-Substituted Phenyl-3-(4-(4-(piperazin-1-ylmethyl)thiazol2-yl)phenyl)ureas $\mathbf{9 a - 0}$

To a solution of piperazine (64 g, $0.748 \mathrm{~mol}$ ) in ethanol was added urea 8a-0 in portions. The reaction mixture was stirred at room temperature for $2 \mathrm{~h}$. The resulting mixture was evaporated in vacuo to remove most of the solvent and poured into $1.5 \mathrm{~L}$ water. The white precipitates was filtered, washed with water and dried to obtain $\mathbf{9 a}-\mathbf{0}$.

1-(3-Fluorophenyl)-3-(4-(4-(piperazin-1-ylmethyl)thiazol-2-yl)phenyl)urea (9a): Yield: 78.1\%; ESI-MS m/z: $412.3[\mathrm{M}+\mathrm{H}]^{+}$. 1-(3,5-Dichlorophenyl)-3-(4-(4-(piperazin-1-ylmethyl)thiazol-2-yl)phenyl)urea (9b): Yield: 79.6\%; ESI-MS m/z: $462.1[\mathrm{M}+\mathrm{H}]^{+}$. 1-(3-Chloro-4-fluorophenyl)-3-(4-(4-(piperazin-1-ylmethyl) thiazol-2-yl)phenyl)urea (9c): Yield: 83.5\%; ESI-MS m/z: $446.2[\mathrm{M}+\mathrm{H}]^{+} .1-(4-($ Trifluoromethyl)phenyl)3-(4-(4-(piperazin-1-ylmethyl)thiazol-2-yl)phenyl)urea (9d): Yield: 81.0\%; ESI-MS m/z: 463.2 [M+H] . 
1-(3-(Trifluoromethyl)phenyl)-3-(4-(4-(piperazin-1-ylmethyl)thiazol-2-yl)phenyl)urea (9e): Yield: 83.4\%; ESI-MS m/z: $463.2[\mathrm{M}+\mathrm{H}]^{+}$. 1-(3-Chlorophenyl)-3-(4-(4-(piperazin-1-ylmethyl)thiazol-2-yl)phenyl)urea (9f): Yield: 77.7\%; ESI-MS m/z: $428.2[\mathrm{M}+\mathrm{H}]^{+}$. 1-(3,4-Dichlorophenyl)-3-(4-(4-(piperazin-1-ylmethyl) thiazol-2-yl)phenyl)urea (9g): Yield: 78.5\%; ESI-MS m/z: $462.1[\mathrm{M}+\mathrm{H}]^{+} .1-(3-($ Trifluoromethoxy)phenyl)3-(4-(4-(piperazin-1-ylmethyl)thiazol-2-yl)phenyl)urea (9h): Yield: 80.9\%; ESI-MS m/z: 478.3 $[\mathrm{M}+\mathrm{H}]^{+}$. 1-phenyl-3-(4-(4-(piperazin-1-ylmethyl)thiazol-2-yl)phenyl)urea (9i): Yield: 83.4\%; ESI-MS $\mathrm{m} / z: 394.2[\mathrm{M}+\mathrm{H}]^{+}$. 1-(2-(Trifluoromethyl)phenyl)-3-(4-(4-(piperazin-1-ylmethyl)thiazol-2-yl)phenyl)urea (9j): Yield: 55.6\%; ESI-MS m/z: $463.2[\mathrm{M}+\mathrm{H}]^{+}$. 1-(4-Chloro-3-(trifluoromethyl)phenyl)-3-(4-(4(piperazin-1-ylmethyl)thiazol-2-yl)phenyl)urea (9k): Yield: 86.8\%; ESI-MS $m / z$ : $480.2[\mathrm{M}+\mathrm{H}]^{+}$. 1-(3Methoxyphenyl)-3-(4-(4-(piperazin-1-ylmethyl)thiazol-2-yl)phenyl)urea (91): Yield: 78.9\%; ESI-MS $m / z: \quad 424.3 \quad[\mathrm{M}+\mathrm{H}]^{+} . \quad 1-(3,5-D i($ trifluoromethyl)phenyl)-3-(4-(4-(piperazin-1-ylmethyl)thiazol-2-yl) phenyl)urea (9m): Yield: 79.6\%; ESI-MS m/z: $530.2[\mathrm{M}+\mathrm{H}]^{+} .1$-3,4-Dimethylphenyl)phenyl)-3-(4-(4(piperazin-1-ylmethyl)thiazol-2-yl)phenyl)urea (9n): Yield: 75.0\%; ESI-MS m/z: $422.2[\mathrm{M}+\mathrm{H}]^{+}$. 1-(3Chloro-4-(trifluoromethyl)phenyl)-3-(4-(4-(piperazin-1-ylmethyl)thiazol-2-yl)phenyl)urea (9o): Yield: 79.4\%; ESI-MS $m / z: 480.2[\mathrm{M}+\mathrm{H}]^{+}$.

3.7. General Procedure for Preparation of Ethyl 2-(4-((2-(4-(3-substituted phenylureido)phenyl)thiazol4-yl)methyl)piperazin-1-yl)acetates 10a-0

To a solution of $N$-substituted piperazine 9a-o $(0.063 \mathrm{~mol})$ in ethanol $(300 \mathrm{~mL})$ was added potassium carbonate $(5.2 \mathrm{~g}, 0.038 \mathrm{~mol})$, ethyl chloroacetate $(7.7 \mathrm{~g}, 0.063 \mathrm{~mol})$ and sodium iodide (cat.). The reaction mixture was heated to $50{ }^{\circ} \mathrm{C}$ and stirred for $2 \mathrm{~h}$. The reaction mixture was concentrated in vacuo and cooled. The product precipitated was filtered off, washed with ethanol and water, and dried to obtain 10a-0.

Ethyl 2-(4-((2-(4-(3-(3-fluorophenyl)ureido)phenyl)thiazol-4-yl)methyl)piperazin-1-yl)acetate (10a): Yield: 87.7\%; ESI-MS m/z: $498.2[\mathrm{M}+\mathrm{H}]^{+}$. Ethyl 2-(4-((2-(4-(3-(3,5-dichlorophenyl)ureido)phenyl) thiazol-4-yl)methyl)piperazin-1-yl)acetate (10b): Yield: 88.3\%; ESI-MS $m / z: 548.2[\mathrm{M}+\mathrm{H}]^{+}$. Ethyl 2(4-((2-(4-(3-(3-chloro-4-fluorophenyl)ureido)phenyl)thiazol-4-yl)methyl)piperazin-1-yl)acetate (10c): Yield: 90.4\%; ESI-MS m/z:532.2 [M+H] ${ }^{+}$. Ethyl 2-(4-((2-(4-(3-(4-(trifluoromethyl)phenyl)ureido)phenyl) thiazol-4-yl)methyl)piperazin-1-yl)acetate (10d): Yield: 93.4\%; ESI-MS m/z: $548.2[\mathrm{M}+\mathrm{H}]^{+}$. Ethyl 2(4-((2-(4-(3-(3-(trifluoromethyl)phenyl)ureido)phenyl)thiazol-4-yl)methyl)piperazin-1-yl)acetate (10e): Yield: 92.0\%; ESI-MS m/z: $548.2[\mathrm{M}+\mathrm{H}]^{+}$. Ethyl 2-(4-((2-(4-(3-(3-chlorophenyl)ureido)phenyl)thiazol4-yl)methyl)piperazin-1-yl)acetate (10f): Yield: 89.1\%; ESI-MS $m / z$ : $514.2[\mathrm{M}+\mathrm{H}]^{+}$. Ethyl 2-(4-((2-(4(3-(3,4-dichlorophenyl)ureido)phenyl)thiazol-4-yl)methyl)piperazin-1-yl)acetate (10g): Yield: 87.0\%; ESI-MS m/z: $548.1[\mathrm{M}+\mathrm{H}]^{+}$. Ethyl 2-(4-((2-(4-(3-(3-(trifluoromethoxy)phenyl)ureido)phenyl)thiazol-4yl)methyl)piperazin-1-yl)acetate (10h): Yield: 88.7\%; ESI-MS m/z: $564.2[\mathrm{M}+\mathrm{H}]^{+}$. Ethyl 2-(4-((2-(4(3-phenylureido)phenyl)thiazol-4-yl)methyl)piperazin-1-yl)acetate (10i): Yield: 90.4\%; ESI-MS $\mathrm{m} / \mathrm{z}$ : $480.2[\mathrm{M}+\mathrm{H}]^{+}$. Ethyl 2-(4-((2-(4-(3-(2-(trifluoromethyl)phenyl)ureido)phenyl)thiazol-4-yl)methyl)piperazin1-yl)acetate (10j): Yield: 91.7\%; ESI-MS m/z: $548.2[\mathrm{M}+\mathrm{H}]^{+}$. Ethyl 2-(4-((2-(4-(3-(4-chloro-3(trifluoromethyl)phenyl)ureido)phenyl)thiazol-4-yl)methyl)piperazin-1-yl)acetate (10k) Yield: 91.5\%; ESI-MS m/z: $582.1[\mathrm{M}+\mathrm{H}]^{+}$. Ethyl 2-(4-((2-(4-(3-(3-methoxyphenyl)ureido)phenyl)thiazol-4-yl)methyl) 
piperazin-1-yl)acetate (101) Yield: 93.4\%; ESI-MS m/z: $498.2[\mathrm{M}+\mathrm{H}]^{+}$. Ethyl 2-(4-((2-(4-(3-(3,5di(trifluoromethyl)phenyl)ureido)phenyl)thiazol-4-yl)methyl)piperazin-1-yl)acetate (10m): Yield: 95.0\%; ESI-MS m/z: $616.2[\mathrm{M}+\mathrm{H}]^{+}$. Ethyl 2-(4-((2-(4-(3-(3,4-dimethylphenyl)ureido)phenyl)thiazol-4-yl)methyl) piperazin-1-yl)acetate (10n) Yield: 88.8\%; ESI-MS m/z: $508.3[\mathrm{M}+\mathrm{H}]^{+}$. Ethyl 2-(4-((2-(4-(3-(3-chloro4-(trifluoromethyl)phenyl)ureido)phenyl)thiazol-4-yl)methyl)piperazin-1-yl)acetate (10o): Yield: 88.4\%; ESI-MS $m / z: 582.1[\mathrm{M}+\mathrm{H}]^{+}$.

3.8. General Procedure for Preparation of 1-Substituted phenyl-3-(4-(4-((4-(2-hydrazinyl-2-oxoethyl) piperazin-1-yl)methyl)thiazol-2-yl)phenyl)ureas 11a-0

To a solution of ester $\mathbf{1 0 a}-\mathbf{o}(0.044 \mathrm{~mol})$ in ethanol $(250 \mathrm{~mL})$ was added $80 \%$ hydrazine hydrate $(27.7 \mathrm{~g}, 0.443 \mathrm{~mol})$. The reaction mixture was heated to $50{ }^{\circ} \mathrm{C}$ and stirred for $48 \mathrm{~h}$. The reaction mixture was evaporated to remove most of the solvent. The residue was filtered off, washed with water, and dried to obtain $\mathbf{1 1 a}-\mathbf{0}$.

1-(3-Fluorophenyl)-3-(4-(4-((4-(2-hydrazinyl-2-oxoethyl)piperazin-1-yl)methyl)thiazol-2-yl)phenyl)urea (11a): Yield: 85.1\%; ESI-MS m/z: $484.2[\mathrm{M}+\mathrm{H}]^{+}$. 1-(3,5-Dichlorophenyl)-3-(4-(4-((4-(2-hydrazinyl-2oxoethyl)piperazin-1-yl)methyl)thiazol-2-yl)phenyl)urea (11b): Yield: 83.7\%; ESI-MS m/z: 534.2 $[\mathrm{M}+\mathrm{H}]^{+}$. 1-(3-Chloro-4-fluorophenyl)-3-(4-(4-((4-(2-hydrazinyl-2-oxoethyl)piperazin-1-yl)methyl)thiazol2-yl)phenyl)urea (11c): Yield: 88.9\%; ESI-MS m/z:518.1 [M+H] $]^{+}$. 1-(4-(Trifluoromethyl)phenyl)-3-(4(4-((4-(2-hydrazinyl-2-oxoethyl)piperazin-1-yl)methyl)thiazol-2-yl)phenyl)urea (11d): Yield: 85.3\%; ESI-MS m/z: $534.1[\mathrm{M}+\mathrm{H}]^{+}$. 1-(3-(Trifluoromethyl)phenyl)-3-(4-(4-((4-(2-hydrazinyl-2-oxoethyl)piperazin1-yl)methyl)thiazol-2-yl)phenyl)urea (11e): Yield: 86.7\%; ESI-MS m/z: $534.1[\mathrm{M}+\mathrm{H}]^{+} .1-(3-C h l o r o p h e n y l)$ -3-(4-(4-((4-(2-hydrazinyl-2-oxoethyl)piperazin-1-yl)methyl)thiazol-2-yl)phenyl)urea (11f): Yield: 85.1\%; ESI-MS $m / z: 500.2[\mathrm{M}+\mathrm{H}]^{+}$. 1-(3,4-Dichlorophenyl)-3-(4-(4-((4-(2-hydrazinyl-2-oxoethyl)piperazin-1yl)methyl)thiazol-2-yl)phenyl)urea (11g): Yield: 87.5\%; $\quad$ ESI-MS $m / z$ : $534.2 \quad[\mathrm{M}+\mathrm{H}]^{+} . \quad 1-(3-$ (Trifluoromethoxy)phenyl)-3-(4-(4-((4-(2-hydrazinyl-2-oxoethyl)piperazin-1-yl)methyl)thiazol-2-yl) phenyl)urea (11h): Yield: 85.4\%; ESI-MS m/z: $550.3[\mathrm{M}+\mathrm{H}]^{+}$. 1-phenyl-3-(4-(4-((4-(2-hydrazinyl-2oxoethyl)piperazin-1-yl)methyl)thiazol-2-yl)phenyl)urea (11i): Yield: 87.8\%; ESI-MS $\mathrm{m} / \mathrm{z}$ : 466.2 $[\mathrm{M}+\mathrm{H}]^{+}$. 1-(2-(Trifluoromethyl)phenyl)-3-(4-(4-((4-(2-hydrazinyl-2-oxoethyl)piperazin-1-yl)methyl)thiazol2-yl)phenyl)urea (11j): Yield: 88.1\%; ESI-MS m/z: $534.1[\mathrm{M}+\mathrm{H}]^{+}$. 1-(4-Chloro-3-(trifluoromethyl) phenyl)-3-(4-(4-((4-(2-hydrazinyl-2-oxoethyl)piperazin-1-yl)methyl)thiazol-2-yl)phenyl)urea (11k): Yield: 88.0\%; ESI-MS m/z: $568.2[\mathrm{M}+\mathrm{H}]^{+}$. 1-(3-Methoxyphenyl)-3-(4-(4-((4-(2-hydrazinyl-2-oxoethyl)piperazin1-yl)methyl)thiazol-2-yl)phenyl)urea (11I): Yield: 85.2\%; ESI-MS $m / z$ : $496.3[\mathrm{M}+\mathrm{H}]^{+} .1-(3,5-$ Di(trifluoromethyl)phenyl)-3-(4-(4-((4-(2-hydrazinyl-2-oxoethyl)piperazin-1-yl)methyl)thiazol-2yl)phenyl)urea (11m): Yield: 84.7\%; ESI-MS m/z: $602.2[\mathrm{M}+\mathrm{H}]^{+} .1-(3,4-D i m e t h y l p h e n y l)-3-(4-(4-((4-$ (2-hydrazinyl-2-oxoethyl)piperazin-1-yl)methyl)thiazol-2-yl)phenyl)urea (11n): Yield: 88.2\%; ESI-MS $\mathrm{m} / \mathrm{z}: 494.3[\mathrm{M}+\mathrm{H}]^{+}$. 1-(3-Chloro-4-(trifluoromethyl)phenyl)-3-(4-(4-((4-(2-hydrazinyl-2-oxoethyl)piperazin1-yl)methyl)thiazol-2-yl)phenyl)urea (110): Yield: 87.34\%; ESI-MS m/z: $568.2[\mathrm{M}+\mathrm{H}]^{+}$. 


\subsection{General Procedure for Preparation of 5-Benzyloxy-2-hydroxybenzaldehydes 12a-d}

To a solution of 2,5-dihydroxybenzaldehyde $(50 \mathrm{~g}, 0.362 \mathrm{~mol})$ in acetonitrile $(500 \mathrm{~mL})$ was added substituted benzyl chloride $(0.471 \mathrm{~mol})$, sodium hydrogen carbonate $(35 \mathrm{~g}, 0.413 \mathrm{~mol})$ and potassium iodide (cat.). The reaction mixture was heated to reflux and stirred for $30 \mathrm{~h}$. The mixture was poured into water $(500 \mathrm{~mL})$. The precipitates were filtered off, dried, recrystallized from methanol to give $12 \mathbf{a}-\mathbf{d}$.

5-(Benzyloxy)-2-hydroxybenzaldehyde (12a): Yield: 53.5\%; $\quad$ ESI-MS $\quad m / z: 227.1 \quad[\mathrm{M}+\mathrm{H}]^{+}$. 5-((2-Fluorobenzyl)oxy)-2-hydroxybenzaldehyde (12b): Yield: 48.8\%; ESI-MS m/z: $245.1[\mathrm{M}+\mathrm{H}]^{+}$. 5-((4-Chlorobenzyl)oxy)-2-hydroxybenzaldehyde (12c): Yield: 45.5\%; ESI-MS m/z:261.0 [M+H] . 5-((3-Chlorobenzyl)oxy)-2-hydroxybenzaldehyde (12d): Yield: 46.9\%; ESI-MS m/z: 261.0 [M+H] ${ }^{+}$.

\subsection{General Procedure for Preparation of 4-Benzyloxy-2-hydroxybenzaldehydes 13a-c}

To a solution of 2,4-dihydroxybenzaldehyde $(50 \mathrm{~g}, 0.362 \mathrm{~mol})$ in acetonitrile $(500 \mathrm{~mL})$ was added benzyl chloride (60 g, $0.471 \mathrm{~mol})$, sodium hydrogencarbonate $(35 \mathrm{~g}, 0.413 \mathrm{~mol})$ and potassium iodide (cat.). The reaction mixture was heated to reflux and stirred for $30 \mathrm{~h}$. The mixture was poured into water $(500 \mathrm{~mL})$. The precipitates were filtered off, dried, recrystallized from methanol to give 13a-c.

4-((4-Chlorobenzyl)oxy)-2-hydroxybenzaldehyde (13a): Yield: 49.1\%; ESI-MS m/z: 260.9 [M+H] . 4-((2,4-Dichlorobenzyl)oxy)-2-hydroxybenzaldehyde (13b): Yield: 50.4\%; ESI-MS m/z: 295.0 [M+H] . 4-((3-Fluorobenzyl)oxy)-2-hydroxybenzaldehyde (13c): Yield: 49.7\%; ESI-MS m/z: $245.1[\mathrm{M}+\mathrm{H}]^{+}$.

\subsection{7-Hydroxy-4-methyl-2H-chromen-2-one (14)}

Sulfuric acid $(500 \mathrm{~mL})$ was cooled below $10{ }^{\circ} \mathrm{C}$ in an ice-salt bath, and to the cooled acid was slowly added a solution of $m$-dihydroxybenzene $(55 \mathrm{~g}, 0.500 \mathrm{~mol})$ in ethyl acetoacetate $(65 \mathrm{~g}, 0.500 \mathrm{~mol})$. The reaction mixture was stirred for $4 \mathrm{~h}$. The resulting mixture was poured onto cracked ice and stirred. The precipitates were filtered off, washed with water and dried to afford $14(67.0 \mathrm{~g}, 76.1 \%)$ as a white solid. ESI-MS $m / z: 175.0[\mathrm{M}+\mathrm{H}]^{+}$.

\subsection{7-Hydroxy-4-methyl-2-oxo-2H-chromene-6-carbaldehyde (15)}

A suspension of urotropine $(20 \mathrm{~g}, 0.143 \mathrm{~mol})$ in glacial acetic acid $(80 \mathrm{~mL})$ was heated to $40{ }^{\circ} \mathrm{C}$ and stirred until a clear solution formed. To this solution was added 14 (5 g, $0.028 \mathrm{~mol})$ in portions. The reaction mixture was stirred for $20 \mathrm{~min}$ and then heated to $115^{\circ} \mathrm{C}$ and stirred for another $2 \mathrm{~h}$. Finally the reaction mixture was cooled to $95{ }^{\circ} \mathrm{C}, 30 \%$ sulfuric acid $(15 \mathrm{~mL})$ was added and the reaction continued for $1.5 \mathrm{~h}$. The resulting mixture was evaporated to dryness and the dry residue obtained was extracted with ether. The organic layer was then washed with saturated sodium hydrogencarbonate solution, dried over anhydrous sodium sulfate and evaporated to dryness to afford $\mathbf{1 5}(2.2 \mathrm{~g}, 38.0 \%)$ as a white solid. ESI-MS m/z: $205.0[\mathrm{M}+\mathrm{H}]^{+}$. 


\subsection{General Procedure for Preparation of Substituted 4-Substituted Phenyl Isocyanates 16a-b}

To a solution of triphosgene $(40 \mathrm{~g}, 0.135 \mathrm{~mol})$ in dioxane $(100 \mathrm{~mL})$ was added substituted aniline $(0.270 \mathrm{~mol})$ dropwise. The reaction mixture was heated to $80^{\circ} \mathrm{C}$ and stirred for $24 \mathrm{~h}$. The reaction mixture was evaporated in vacuo to remove the solvent. The residue was distilled under reduced pressure to give the desired phenyl isocyanates $\mathbf{1 6} \mathbf{a}-\mathbf{b}$.

1-Isocyanato-4-(trifluoromethyl)benzene (16a): Yield: $70.6 \%$; b.p.: $90-92 \quad{ }^{\circ} \mathrm{C} \quad(20 \mathrm{mmHg})$. 1-Fluoro-4-isocyanatobenzene (16b): Yield: 73.1\%; b.p.: $75-77^{\circ} \mathrm{C}(20 \mathrm{mmHg})$.

\subsection{General Procedure for Preparation of Methyl 2-(3-(4-Substituted phenyl)ureido)acetates 17a-b}

To a suspension of glycine methyl ester hydrochloride $(16 \mathrm{~g}, 0.130 \mathrm{~mol})$ in dichloromethane $(150 \mathrm{~mL})$ was added triethylamine $(15.6 \mathrm{~g}, 0.154 \mathrm{~mol})$ dropwise. The mixture was stirred at room temperature for $1 \mathrm{~h}$ and cooled below $0{ }^{\circ} \mathrm{C}$ in an ice bath. To the cold mixture was added isocyanate 16a-b $(0.118 \mathrm{~mol})$ slowly. The reaction mixture was kept in an ice bath and stirred for $4 \mathrm{~h}$, then another $1 \mathrm{~h}$ at room temperature. The reaction mixture was evaporated to dryness, poured into water $(300 \mathrm{~mL})$ and stirred. The precipitates were collected by filtration and dried to give $\mathbf{1 7} \mathbf{a}-\mathbf{b}$.

Methyl 2-(3-(4-(trifluoromethyl)phenyl)ureido)acetate (17a): Yield: 80.6\%; ESI-MS m/z: $277.1[\mathrm{M}+\mathrm{H}]^{+}$. Methyl 2-(3-(4-fluorophenyl)ureido)acetate (17b): Yield: 77.8\%; ESI-MS m/z: $227.1[\mathrm{M}+\mathrm{H}]^{+}$.

\subsection{General Procedure for Preparation of 3-(4-Substituted phenyl)imidazolidine-2,4-diones 18a-b}

To a solution of ester $\mathbf{1 7 a}-\mathbf{b}(0.044 \mathrm{~mol})$ in acetone $(100 \mathrm{~mL})$ was added concentrated hydrochloride $(110 \mathrm{~mL})$. The reaction mixture was stirred and refluxed for $4 \mathrm{~h}$. The reaction mixture was evaporated to remove most of the solvent and cooled. The precipitates were filtered off, washed by water and dried to give $\mathbf{1 8 a}-\mathbf{b}$.

3-(4-(Trifluoromethyl)phenyl)imidazolidine-2,4-dione (18a): Yield: 66.7\%; ESI-MS m/z: $245.1[\mathrm{M}+\mathrm{H}]^{+}$. 3-(4-Fluorophenyl)imidazolidine-2,4-dione (18b): Yield: 61.2\%; ESI-MS m/z: $195.1[\mathrm{M}+\mathrm{H}]^{+}$.

\subsection{General Procedure for Preparation of 5-((dimethylamino)methylene)-3-(4-substituted phenyl)} imidazolidine-2,4-diones 19a-b

Imidazolindione 18a-b (0.026 mol), N,N-dimethylformamide dimethylacetal (12 g, $0.103 \mathrm{~mol})$ were added to acetonitrile $(10 \mathrm{~mL})$. The reaction mixture was stirred and refluxed for $4 \mathrm{~h}$. The reaction mixture was cooled, and precipitates were filtered off, washed by acetonitrile in small portions, dried to give 19a-b.

5-((Dimethylamino)methylene)-3-(4-(trifluoromethyl)phenyl)imidazolidine-2,4-dione (19a) Yield: 47.7\%; ESI-MS m/z: $277.1[\mathrm{M}+\mathrm{H}]^{+}$. 5-((Dimethylamino)methylene)-3-(4-fluorophenyl)imidazolidine-2,4-dione (19b): Yield: 52.4\%; ESI-MS m/z: $227.1[\mathrm{M}+\mathrm{H}]^{+}$. 


\subsection{General Procedure for the Preparation of Target Compounds $\mathbf{1 a}-\mathbf{g}, \mathbf{2 a}-\mathbf{k}$, and $\mathbf{3 a}-\mathbf{e}$}

To a solution of acethydrazide $11 \mathbf{a}-\mathbf{0}(0.002 \mathrm{~mol})$ in ethanol $(10 \mathrm{~mL})$ was added appropriate benzaldehyde or the prepared aromatic aldehyde $12 \mathbf{a}-\mathbf{d}, \mathbf{1 3 a}-\mathbf{c}$ or 15 . The reaction mixture was stirred and refluxed for $2 \mathrm{~h}$. The reaction mixture was cooled and precipitates were collected by filtration to obtain the crude product, which was then purified by flash column chromatography. The pure product was dissolved in chloroform. To the solution was added excess hydrogen chloride-ethanol and stirred for $1 \mathrm{~h}$. Ether was added to the mixture above. The precipitates were filtered off and dried to afford $\mathbf{1 a}-\mathbf{g}, \mathbf{2} \mathbf{a}-\mathbf{k}$, and $\mathbf{3 a}-\mathbf{e}$ as dihydrochlorides.

1-(3-Fluorophenyl)-3-(4-(4-((4-(2-(2-(2-hydroxy-4-methylbenzylidene)hydrazinyl)-2-oxoethyl)piperazin1-yl)methyl)thiazol-2-yl)phenyl)urea (1a). Yield: 80.1\%; M.p.: 185-187 ${ }^{\circ} \mathrm{C}$; ESI-MS m/z: 602.1 $[\mathrm{M}-2 \mathrm{HCl}+\mathrm{H}]^{+} ;{ }^{1} \mathrm{H}-\mathrm{NMR}\left(\mathrm{DMSO}-d_{6}\right) \delta(\mathrm{ppm}): 11.66(\mathrm{~s}, 1 \mathrm{H}), 11.50(\mathrm{~s}, 1 \mathrm{H}), 10.44(\mathrm{~s}, 1 \mathrm{H}), 9.03$ $(\mathrm{s}, 1 \mathrm{H}), 8.97(\mathrm{~s}, 1 \mathrm{H}), 8.42(\mathrm{~s}, 1 \mathrm{H}), 7.86(\mathrm{~d}, 2 \mathrm{H}), 7.58(\mathrm{~d}, 2 \mathrm{H}), 7.50(\mathrm{~d}, 1 \mathrm{H}), 7.41(\mathrm{~s}, 1 \mathrm{H}), 7.32(\mathrm{dd}, 1 \mathrm{H})$, $7.15(\mathrm{~d}, 1 \mathrm{H}), 7.04(\mathrm{~s}, 1 \mathrm{H}), 6.98(\mathrm{~s}, 1 \mathrm{H}), 6.80(\mathrm{t}, 1 \mathrm{H}), 3.66(\mathrm{~s}, 2 \mathrm{H}), 3.34(\mathrm{~s}, 1 \mathrm{H}), 3.32(\mathrm{~s}, 2 \mathrm{H}), 3.12$ (s, 1H), $2.54(\mathrm{~s}, 6 \mathrm{H}), 2.22(\mathrm{~s}, 3 \mathrm{H})$. Anal. Calcd for $\mathrm{C}_{31} \mathrm{H}_{32} \mathrm{FN}_{7} \mathrm{O}_{3} \mathrm{~S}(\%): \mathrm{C}, 61.88 ; \mathrm{H}, 5.36 ; \mathrm{N}, 16.30$; Found (\%): C, 61.81; H, 5.42; N, 16.28.

1-(3,5-Dichlorophenyl)-3-(4-(4-((4-(2-(2-(2-hydroxy-3-(2-methylallyl)benzylidene)hydrazinyl)-2-oxoethyl) piperazin-1-yl)methyl)thiazol-2-yl)phenyl)urea (1b). Yield: 76.1\%; M.p.: $178-180{ }^{\circ} \mathrm{C}$; ESI-MS $\mathrm{m} / z$ : $691.7[\mathrm{M}-2 \mathrm{HCl}+\mathrm{H}]^{+} ;{ }^{1} \mathrm{H}-\mathrm{NMR}\left(\mathrm{DMSO}-d_{6}\right) \delta(\mathrm{ppm}): 11.89(\mathrm{~s}, 1 \mathrm{H}), 11.50(\mathrm{~s}, 1 \mathrm{H}), 9.20(\mathrm{~s}, 1 \mathrm{H}), 9.15$ (s, 1H), $8.49(\mathrm{~s}, 1 \mathrm{H}), 7.86(\mathrm{~m}, 3 \mathrm{H}), 7.57(\mathrm{~m}, 3 \mathrm{H}), 7.41(\mathrm{~s}, 1 \mathrm{H}), 7.25(\mathrm{~d}, 1 \mathrm{H}), 7.18-7.14(\mathrm{~m}, 2 \mathrm{H}), 6.88$ $(\mathrm{t}, 1 \mathrm{H}), 4.75(\mathrm{~s}, 1 \mathrm{H}), 4.63(\mathrm{~s}, 1 \mathrm{H}), 3.66-3.58(\mathrm{~m}, 4 \mathrm{H}), 3.13(\mathrm{~s}, 2 \mathrm{H}), 2.54(\mathrm{~s}, 8 \mathrm{H}), 1.67(\mathrm{~s}, 3 \mathrm{H})$. Anal. Calcd for $\mathrm{C}_{34} \mathrm{H}_{35} \mathrm{Cl}_{2} \mathrm{~N}_{7} \mathrm{O}_{3} \mathrm{~S}(\%)$ : C, 58.96; H, 5.09; N, 14.16; Found (\%): C, 58.94; H, 5.02; N, 14.22.

1-(3-Chloro-4-fluorophenyl)-3-(4-(4-((4-(2-(2-(3,5-di-tert-butyl-2-hydroxybenzylidene)hydrazinyl)-2oxoethyl)piperazin-1-yl)methyl)thiazol-2-yl)phenyl)urea (1c). Yield: 82.7\%; M.p.: 231-232 ${ }^{\circ} \mathrm{C}$; ESI-MS $m / z: 734.6[\mathrm{M}-2 \mathrm{HCl}+\mathrm{H}]^{+} ;{ }^{1} \mathrm{H}-\mathrm{NMR}\left(\mathrm{DMSO}-d_{6}\right) \delta(\mathrm{ppm}): 11.98(\mathrm{~s}, 1 \mathrm{H}), 9.71(\mathrm{~s}, 1 \mathrm{H}), 9.67(\mathrm{~s}, 1 \mathrm{H})$, $9.09(\mathrm{~s}, 1 \mathrm{H}), 8.47(\mathrm{~s}, 1 \mathrm{H}), 7.91(\mathrm{~d}, 3 \mathrm{H}), 7.81(\mathrm{~d}, 1 \mathrm{H}), 7.62(\mathrm{~d}, 2 \mathrm{H}), 7.44(\mathrm{~d}, 1 \mathrm{H}), 7.38-7.33(\mathrm{~m}, 2 \mathrm{H})$, $7.22(\mathrm{~s}, 1 \mathrm{H}), 4.45(\mathrm{~s}, 2 \mathrm{H}), 3.67$ (brs, $10 \mathrm{H}), 1.41(\mathrm{~d}, 9 \mathrm{H}), 1.28(\mathrm{~d}, 9 \mathrm{H})$. Anal. Calcd for $\mathrm{C}_{38} \mathrm{H}_{45} \mathrm{ClFN}_{7} \mathrm{O}_{3} \mathrm{~S}$ (\%):C, 62.15; H, 6.18; N, 13.35; Found (\%): C, 62.17; H, 6.11; N, 13.28.

1-(4-(4-((4-(2-(2-(2-Hydroxy-6-isopropyl-3-methylbenzylidene)hydrazinyl)-2-oxoethyl)piperazin-1-yl) methyl)thiazol-2-yl)phenyl)-3-(4-(trifluoromethyl)phenyl)urea (1d). Yield: 77.4\%; M.p.: 185-187 ${ }^{\circ} \mathrm{C}$; ESI-MS m/z: $694.5[\mathrm{M}-2 \mathrm{HCl}+\mathrm{H}]^{+} .{ }^{1} \mathrm{H}-\mathrm{NMR}\left(\mathrm{DMSO}-d_{6}\right) \delta(\mathrm{ppm}): 11.98(\mathrm{~s}, 1 \mathrm{H}), 10.14(\mathrm{~s}, 1 \mathrm{H}), 10.05$ $(\mathrm{s}, 1 \mathrm{H}), 9.01(\mathrm{~s}, 1 \mathrm{H}), 8.54(\mathrm{~s}, 1 \mathrm{H}), 8.37(\mathrm{~s}, 1 \mathrm{H}), 7.98-7.93(\mathrm{~m}, 3 \mathrm{H}), 7.70-7.64(\mathrm{~m}, 6 \mathrm{H}), 7.33(\mathrm{t}, 1 \mathrm{H})$, $6.90(\mathrm{dd}, 1 \mathrm{H}), 4.67$ (s, 2H), $4.56(\mathrm{~s}, 2 \mathrm{H}), 3.60$ (brs, 8H), $1.28(\mathrm{~d}, 9 \mathrm{H})$. Anal. Calcd for $\mathrm{C}_{35} \mathrm{H}_{38} \mathrm{~F}_{3} \mathrm{~N}_{7} \mathrm{O}_{3} \mathrm{~S}$ (\%):C, 60.59; H, 5.52; N, 14.13; Found (\%): C, 60.61; H, 5.51; N, 14.18.

1-(4-(4-((4-(2-(2-(5-(tert-Butyl)-2-hydroxybenzylidene)hydrazinyl)-2-oxoethyl)piperazin-1-yl)methyl) thiazol-2-yl)phenyl)-3-(4-(trifluoromethyl)phenyl)urea (1e). Yield: 75.1\%; M.p.: 215-218 ${ }^{\circ} \mathrm{C}$; ESI-MS $m / z: 694.4[\mathrm{M}-2 \mathrm{HCl}+\mathrm{H}]^{+} ;{ }^{1} \mathrm{H}-\mathrm{NMR}\left(\mathrm{DMSO}-d_{6}\right) \delta(\mathrm{ppm}): 12.51(\mathrm{~s}, 1 \mathrm{H}), 11.70(\mathrm{~s}, 1 \mathrm{H}), 10.00(\mathrm{~s}, 1 \mathrm{H})$, $9.95(\mathrm{~s}, 1 \mathrm{H}), 8.90(\mathrm{~s}, 1 \mathrm{H}), 7.89(\mathrm{~s}, 3 \mathrm{H}), 7.75-7.62(\mathrm{~m}, 7 \mathrm{H}), 7.14(\mathrm{~d}, 1 \mathrm{H}), 6.75(\mathrm{~d}, 1 \mathrm{H}), 4.27-4.09$ 
(m, 2H), $3.35(\mathrm{~s}, 1 \mathrm{H}), 3.17(\mathrm{~s}, 4 \mathrm{H}), 3.07(\mathrm{~s}, 2 \mathrm{H}), 2.87(\mathrm{~s}, 4 \mathrm{H}), 2.13(\mathrm{~s}, 3 \mathrm{H}), 1.21(\mathrm{~s}, 6 \mathrm{H})$. Anal. Calcd for $\mathrm{C}_{35} \mathrm{H}_{38} \mathrm{~F}_{3} \mathrm{~N}_{7} \mathrm{O}_{3} \mathrm{~S}(\%): \mathrm{C}, 60.59 ; \mathrm{H}, 5.52 ; \mathrm{N}, 14.13$; Found (\%): C, 60.51; H, 5.48; N, 14.16.

1-(4-(4-((4-(2-(2-(2-Hydroxy-5-methoxybenzylidene)hydrazinyl)-2-oxoethyl)piperazin-1-yl)methyl)thiazol2-yl)phenyl)-3-(4-(trifluoromethyl)phenyl)urea (1f). Yield: 70.7\%; M.p.: 198-200 ${ }^{\circ} \mathrm{C}$; ESI-MS $\mathrm{m} / z$ : $667.9[\mathrm{M}-2 \mathrm{HCl}+\mathrm{H}]^{+} ;{ }^{1} \mathrm{H}-\mathrm{NMR}\left(\mathrm{DMSO}-d_{6}\right) \delta(\mathrm{ppm}): 12.00(\mathrm{~s}, 1 \mathrm{H}), 10.22(\mathrm{~s}, 1 \mathrm{H}), 10.12(\mathrm{~s}, 1 \mathrm{H}), 8.52$ $(\mathrm{s}, 1 \mathrm{H}), 8.35(\mathrm{~s}, 1 \mathrm{H}), 7.98-7.92(\mathrm{~m}, 4 \mathrm{H}), 7.70-7.62(\mathrm{~m}, 7 \mathrm{H}), 7.28(\mathrm{~s}, 1 \mathrm{H}), 6.89(\mathrm{~s}, 2 \mathrm{H}), 4.67(\mathrm{~s}, 1 \mathrm{H})$, 4.57 (s, 2H), 3.72 (s, 3H), 3.64 (brs, $9 \mathrm{H})$. Anal. Calcd for $\mathrm{C}_{32} \mathrm{H}_{33} \mathrm{~F}_{3} \mathrm{~N}_{7} \mathrm{O}_{4} \mathrm{~S}(\%): \mathrm{C}, 57.56 ; \mathrm{H}, 4.83 ; \mathrm{N}$, 14.68; Found (\%): C, 57.53; H, 4.85; N, 14.71.

1-(4-(4-((4-(2-(2-(2-Hydroxybenzylidene)hydrazinyl)-2-oxoethyl)piperazin-1-yl)methyl)thiazol-2-yl) phenyl)-3-(3-(trifluoromethyl)phenyl)urea (1g). Yield: 78.4\%; M.p.: 205-207 ${ }^{\circ} \mathrm{C}$; ESI-MS m/z: 638.1 $[\mathrm{M}-2 \mathrm{HCl}+\mathrm{H}]^{+} ;{ }^{1} \mathrm{H}-\mathrm{NMR}\left(\mathrm{DMSO}-d_{6}\right) \delta(\mathrm{ppm}): 11.94(\mathrm{~s}, 1 \mathrm{H}), 10.14(\mathrm{~s}, 1 \mathrm{H}), 9.84(\mathrm{~s}, 1 \mathrm{H}), 9.74(\mathrm{~d}, 1 \mathrm{H})$, $8.51(\mathrm{~s}, 1 \mathrm{H}), 8.36(\mathrm{~s}, 1 \mathrm{H}), 8.02(\mathrm{~s}, 1 \mathrm{H}), 7.93(\mathrm{~d}, 2 \mathrm{H}), 7.89-7.88(\mathrm{~m}, 1 \mathrm{H}), 7.75(\mathrm{~d}, 1 \mathrm{H}), 7.66-7.50(\mathrm{~m}, 4 \mathrm{H})$, 7.34-7.24 (m, 2H), 6.94-6.84 (m, 2H), 4.54 (s, 1H), 4.45 (s, 1H), 4.03 (s, 2H), 3.51 (brs, 8H). Anal. Calcd for $\mathrm{C}_{31} \mathrm{H}_{30} \mathrm{~F}_{3} \mathrm{~N}_{7} \mathrm{O}_{3} \mathrm{~S}(\%): \mathrm{C}, 58.39 ; \mathrm{H}, 4.74 ; \mathrm{N}, 15.38$; Found (\%): C, 58.36; H, 4.75; N, 15.36 .

1-(4-(4-((4-(2-(2-(5-(Benzyloxy)-2-hydroxybenzylidene)hydrazinyl)-2-oxoethyl)piperazin-1-yl)methyl) thiazol-2-yl)phenyl)-3-(3-fluorophenyl)urea (2a). Yield: 74.9\%; M.p.: 190-192 ${ }^{\circ} \mathrm{C}$; ESI-MS m/z: 694.5 $[\mathrm{M}-2 \mathrm{HCl}+\mathrm{H}]^{+} ;{ }^{1} \mathrm{H}-\mathrm{NMR}\left(\mathrm{DMSO}-d_{6}\right) \delta(\mathrm{ppm}): 11.41(\mathrm{~s}, 1 \mathrm{H}), 10.66(\mathrm{~s}, 1 \mathrm{H}), 9.72(\mathrm{~s}, 1 \mathrm{H}), 9.09(\mathrm{~s}, 1 \mathrm{H})$, $9.03(\mathrm{~s}, 1 \mathrm{H}), 8.47(\mathrm{~s}, 1 \mathrm{H}), 7.85(\mathrm{~d}, 2 \mathrm{H}), 7.59(\mathrm{~d}, 2 \mathrm{H}), 7.50(\mathrm{~d}, 1 \mathrm{H}), 7.47-7.25(\mathrm{~m}, 7 \mathrm{H}), 7.23-7.08(\mathrm{~m}$, 2H), $6.97(\mathrm{dd}, 1 \mathrm{H}), 6.85-6.77(\mathrm{~m}, 2 \mathrm{H}), 5.04(\mathrm{~s}, 2 \mathrm{H}), 3.64(\mathrm{~d}, 2 \mathrm{H}), 3.31(\mathrm{~s}, 4 \mathrm{H}), 2.52(\mathrm{~s}, 6 \mathrm{H})$. Anal. Calcd for $\mathrm{C}_{37} \mathrm{H}_{36} \mathrm{FN}_{7} \mathrm{O}_{4} \mathrm{~S}(\%)$ : C, 64.05; H, 5.23; N, 14.13; Found (\%): C, 64.02; H,5.24; N, 14.09 .

1-(4-(4-((4-(2-(2-(5-(Benzyloxy)-2-hydroxybenzylidene)hydrazinyl)-2-oxoethyl)piperazin-1-yl)methyl) thiazol-2-yl)phenyl)-3-(3-chlorophenyl)urea (2b). Yield: 78.3\%; M.p.: 188-190 ${ }^{\circ} \mathrm{C}$; ESI-MS m/z: 709.9 $[\mathrm{M}-2 \mathrm{HCl}+\mathrm{H}]^{+} ;{ }^{1} \mathrm{H}-\mathrm{NMR}\left(\mathrm{DMSO}-d_{6}\right) \delta(\mathrm{ppm}): 11.45(\mathrm{~s}, 1 \mathrm{H}), 10.67(\mathrm{~s}, 1 \mathrm{H}), 9.53(\mathrm{~d}, 2 \mathrm{H}), 8.48(\mathrm{~s}, 1 \mathrm{H})$, $8.17(\mathrm{~s}, 1 \mathrm{H}), 7.85(\mathrm{~d}, 2 \mathrm{H}), 7.72(\mathrm{~s}, 1 \mathrm{H}), 7.59(\mathrm{~d}, 2 \mathrm{H}), 7.45-7.30(\mathrm{~m}, 9 \mathrm{H}), 7.16(\mathrm{dd}, 1 \mathrm{H}), 7.04-6.90(\mathrm{~m}$, $2 \mathrm{H}), 6.86-6.80(\mathrm{~m}, 1 \mathrm{H}), 5.04(\mathrm{~s}, 2 \mathrm{H}), 3.64(\mathrm{~d}, 2 \mathrm{H}), 3.32(\mathrm{~s}, 4 \mathrm{H}), 2.54(\mathrm{~s}, 6 \mathrm{H})$. Anal. Calcd for $\mathrm{C}_{37} \mathrm{H}_{36} \mathrm{ClN}_{7} \mathrm{O}_{4} \mathrm{~S}(\%): \mathrm{C}, 62.57 ; \mathrm{H}, 5.11 ; \mathrm{N}, 13.80$; Found (\%): C, 62.55; H,5.14; N, 13.73.

1-(4-(4-((4-(2-(2-(4-((4-Chlorobenzyl)oxy)-2-hydroxybenzylidene)hydrazinyl)-2-oxoethyl)piperazin-1-yl) methyl)thiazol-2-yl)phenyl)-3-(3-chlorophenyl)urea (2c). Yield: 84.4\%; M.p.: 218-220 ${ }^{\circ} \mathrm{C}$; ESI-MS $m / z: 744.1[\mathrm{M}-2 \mathrm{HCl}+\mathrm{H}]^{+} ;{ }^{1} \mathrm{H}-\mathrm{NMR}\left(\mathrm{DMSO}-d_{6}\right) \delta(\mathrm{ppm}): 11.80(\mathrm{~s}, 1 \mathrm{H}), 9.76(\mathrm{~s}, 1 \mathrm{H}), 9.72(\mathrm{~s}, 1 \mathrm{H})$, $9.67(\mathrm{~s}, 1 \mathrm{H}), 8.41(\mathrm{~s}, 1 \mathrm{H}), 8.26(\mathrm{~s}, 1 \mathrm{H}), 7.93(\mathrm{~s}, 1 \mathrm{H}), 7.90(\mathrm{~s}, 2 \mathrm{H}), 7.71(\mathrm{~s}, 1 \mathrm{H}), 7.63(\mathrm{~d}, 2 \mathrm{H}), 7.46(\mathrm{~s}$, $4 \mathrm{H}), 7.31(\mathrm{~s}, 1 \mathrm{H}), 7.30(\mathrm{~s}, 1 \mathrm{H}), 7.02-7.01(\mathrm{~m}, 1 \mathrm{H}), 6.59-6.54(\mathrm{~m}, 2 \mathrm{H}), 5.11(\mathrm{~d}, 2 \mathrm{H}), 4.51(\mathrm{~s}, 1 \mathrm{H}), 4.45$ (s, 2H), 3.58 (brs, 9H). Anal. Calcd for $\mathrm{C}_{37} \mathrm{H}_{35} \mathrm{Cl}_{2} \mathrm{~N}_{7} \mathrm{O}_{4} \mathrm{~S}$ (\%):C, 59.68; H, 4.74; N, 13.17; Found (\%): C, 59.64; H, 4.70; N, 13.13 .

1-(3-Chlorophenyl)-3-(4-(4-((4-(2-(2-(4-((2,4-dichlorobenzyl)oxy)-2-hydroxybenzylidene)hydrazinyl)2-oxoethyl)piperazin-1-yl)methyl)thiazol-2-yl)phenyl)urea (2d). Yield: 75.2\%; M.p.: 218-220 ${ }^{\circ} \mathrm{C}$; ESI-MS $m / z: 778.0[\mathrm{M}-2 \mathrm{HCl}+\mathrm{H}]^{+} ;{ }^{1} \mathrm{H}-\mathrm{NMR}\left(\mathrm{DMSO}-d_{6}\right) \delta(\mathrm{ppm}): 11.82(\mathrm{~s}, 1 \mathrm{H}), 9.78(\mathrm{~s}, 1 \mathrm{H}), 9.74$ $(\mathrm{s}, 1 \mathrm{H}), 9.70(\mathrm{~s}, 1 \mathrm{H}), 8.27(\mathrm{~s}, 1 \mathrm{H}), 7.94(\mathrm{~s}, 2 \mathrm{H}), 7.91(\mathrm{~s}, 2 \mathrm{H}), 7.71-7.70(\mathrm{~m}, 3 \mathrm{H}), 7.66-7.59(\mathrm{~m}, 4 \mathrm{H})$, $7.50-7.45(\mathrm{~m}, 1 \mathrm{H}), 7.31(\mathrm{~s}, 1 \mathrm{H}), 7.30(\mathrm{~s}, 1 \mathrm{H}), 7.04-7.01(\mathrm{~m}, 1 \mathrm{H}), 6.61-6.56(\mathrm{~m}, 2 \mathrm{H}), 5.15(\mathrm{~d}, 2 \mathrm{H})$, 
$4.53(\mathrm{~s}, 1 \mathrm{H}), 4.46(\mathrm{~s}, 2 \mathrm{H}), 3.66$ (brs, $9 \mathrm{H})$. Anal. Calcd for $\mathrm{C}_{37} \mathrm{H}_{34} \mathrm{Cl}_{3} \mathrm{~N}_{7} \mathrm{O}_{4} \mathrm{~S}(\%)$ : C, 57.04; $\mathrm{H}, 4.40 ; \mathrm{N}$, 12.58; Found (\%): C, 57.06; H,4.38; N, 12.51 .

1-(3,4-Dichlorophenyl)-3-(4-(4-((4-(2-(2-(4-((3-fluorobenzyl)oxy)-2-hydroxybenzylidene)hydrazinyl)-2oxoethyl)piperazin-1-yl)methyl)thiazol-2-yl)phenyl)urea (2e). Yield: 79.4\%; M.p.: 209-210 ${ }^{\circ} \mathrm{C}$; ESI-MS $m / z: 761.8[\mathrm{M}-2 \mathrm{HCl}+\mathrm{H}]^{+} ;{ }^{1} \mathrm{H}-\mathrm{NMR}\left(\mathrm{DMSO}-d_{6}\right) \delta(\mathrm{ppm}): 11.79(\mathrm{~s}, 1 \mathrm{H}), 9.81(\mathrm{~s}, 1 \mathrm{H}), 9.77(\mathrm{~s}, 1 \mathrm{H})$, $9.74(\mathrm{~s}, 1 \mathrm{H}), 9.70(\mathrm{~s}, 1 \mathrm{H}), 8.26(\mathrm{~s}, 1 \mathrm{H}), 7.93-7.88(\mathrm{~m}, 4 \mathrm{H}), 7.62(\mathrm{~d}, 3 \mathrm{H}), 7.53(\mathrm{~d}, 1 \mathrm{H}), 7.46-7.41(\mathrm{~m}, 1 \mathrm{H})$, 7.35 (dd, 1H), 7.29-7.25 (m, 2H), 7.20-7.14 (m, 1H), 6.61-6.55 (m, 2H), $5.15(\mathrm{~s}, 1 \mathrm{H}), 5.12(\mathrm{~s}, 1 \mathrm{H})$, $4.50(\mathrm{~s}, 1 \mathrm{H}), 4.45(\mathrm{~s}, 2 \mathrm{H}), 3.52$ (brs, $9 \mathrm{H})$. Anal. Calcd for $\mathrm{C}_{37} \mathrm{H}_{34} \mathrm{Cl}_{2} \mathrm{FN}_{7} \mathrm{O}_{4} \mathrm{~S}(\%): \mathrm{C}, 58.27 ; \mathrm{H}, 4.49 ; \mathrm{N}$, 12.86; Found (\%): C, 58.26; H,4.47; N, 12.89.

1-(4-(4-((4-(2-(2-(4-((4-Chlorobenzyl)oxy)-2-hydroxybenzylidene)hydrazinyl)-2-oxoethyl)piperazin-1-yl) methyl)thiazol-2-yl)phenyl)-3-(3,4-dichlorophenyl)urea (2f). Yield: 73.5\%; M.p.: 227-228 ${ }^{\circ} \mathrm{C}$; ESI-MS $m / z: 777.7[\mathrm{M}-2 \mathrm{HCl}+\mathrm{H}]^{+} ;{ }^{1} \mathrm{H}-\mathrm{NMR}\left(\mathrm{DMSO}-d_{6}\right) \delta(\mathrm{ppm}): 11.78(\mathrm{~s}, 1 \mathrm{H}), 9.79(\mathrm{~s}, 1 \mathrm{H}), 9.75(\mathrm{~s}, 1 \mathrm{H})$, $9.72(\mathrm{~s}, 1 \mathrm{H}), 9.69(\mathrm{~s}, 1 \mathrm{H}), 8.41(\mathrm{~s}, 1 \mathrm{H}), 8.25(\mathrm{~s}, 1 \mathrm{H}), 7.93-7.88(\mathrm{~m}, 4 \mathrm{H}), 7.62(\mathrm{~d}, 2 \mathrm{H}), 7.53(\mathrm{~d}, 2 \mathrm{H})$, $7.46(\mathrm{~s}, 4 \mathrm{H}), 7.35(\mathrm{dd}, 1 \mathrm{H}), 6.59-6.53(\mathrm{~m}, 2 \mathrm{H}), 5.12(\mathrm{~s}, 1 \mathrm{H}), 5.09(\mathrm{~s}, 1 \mathrm{H}), 4.49(\mathrm{~s}, 1 \mathrm{H}), 4.43(\mathrm{~s}, 2 \mathrm{H})$, 3.53 (s, 9H). Anal. Calcd for $\mathrm{C}_{37} \mathrm{H}_{34} \mathrm{Cl}_{3} \mathrm{~N}_{7} \mathrm{O}_{4} \mathrm{~S}(\%): \mathrm{C}, 57.04 ; \mathrm{H}, 4.40 ; \mathrm{N}, 12.58$; Found (\%): C, 57.06; H,4.47; N, 12.59 .

1-(4-(4-((4-(2-(2-(5-((2-Fluorobenzyl)oxy)-2-hydroxybenzylidene)hydrazinyl)-2-oxoethyl)piperazin-1-yl) methyl)thiazol-2-yl)phenyl)-3-(3-(trifluoromethoxy)phenyl)urea (2g). Yield: 71.2\%; M.p.: 165-168 ${ }^{\circ} \mathrm{C}$; ESI-MS $m / z$ : $778.5[\mathrm{M}-2 \mathrm{HCl}+\mathrm{H}]{ }^{+} ;{ }^{1} \mathrm{H}-\mathrm{NMR}\left(\mathrm{DMSO}-d_{6}\right) \delta(\mathrm{ppm}): 11.42(\mathrm{~s}, 1 \mathrm{H}), 10.71(\mathrm{~s}, 1 \mathrm{H}), 9.75(\mathrm{~s}$, $1 \mathrm{H}), 9.09(\mathrm{~s}, 1 \mathrm{H}), 9.08(\mathrm{~s}, 1 \mathrm{H}), 8.48(\mathrm{~s}, 1 \mathrm{H}), 8.17(\mathrm{~s}, 1 \mathrm{H}), 7.86(\mathrm{~d}, 2 \mathrm{H}), 7.71(\mathrm{~s}, 1 \mathrm{H}), 7.59(\mathrm{~d}, 2 \mathrm{H}), 7.54$ (d, 1H), 7.44-7.39 (m, 3H), 7.33-7.14 (m, 3H), 7.01-6.95 (m, 2H), $6.85(\mathrm{~d}, 1 \mathrm{H}), 5.08(\mathrm{~s}, 2 \mathrm{H}), 3.66(\mathrm{~s}$, $2 \mathrm{H}), 3.47(\mathrm{~s}, 1 \mathrm{H}), 3.10(\mathrm{~s}, 1 \mathrm{H}), 2.54(\mathrm{~s}, 8 \mathrm{H})$. Anal. Calcd for $\mathrm{C}_{38} \mathrm{H}_{35} \mathrm{~F}_{4} \mathrm{~N}_{7} \mathrm{O}_{5} \mathrm{~S}(\%)$ : C, 58.68; H, 4.54; N, 12.61 ; Found (\%): C, 58.66; H,4.59; N, 12.58 .

1-(4-(4-((4-(2-(2-(5-((2-Fluorobenzyl)oxy)-2-hydroxybenzylidene)hydrazinyl)-2-oxoethyl)piperazin-1-yl) methyl)thiazol-2-yl)phenyl)-3-phenylurea (2h). Yield: 78.4\%; M.p.: 202-203 ${ }^{\circ} \mathrm{C}$; ESI-MS m/z: 694.4 $[\mathrm{M}-2 \mathrm{HCl}+\mathrm{H}]^{+} ;{ }^{1} \mathrm{H}-\mathrm{NMR}\left(\mathrm{DMSO}-d_{6}\right) \delta(\mathrm{ppm}): 11.40(\mathrm{~s}, 1 \mathrm{H}), 10.70(\mathrm{~s}, 1 \mathrm{H}), 9.74(\mathrm{~s}, 1 \mathrm{H}), 8.96(\mathrm{~s}, 1 \mathrm{H})$, $8.74(\mathrm{~s}, 1 \mathrm{H}), 8.48(\mathrm{~s}, 1 \mathrm{H}), 7.84(\mathrm{~d}, 2 \mathrm{H}), 7.59-7.52(\mathrm{~m}, 3 \mathrm{H}), 7.47(\mathrm{~d}, 2 \mathrm{H}), 7.40(\mathrm{~s}, 2 \mathrm{H}), 7.32-7.20$ (m, 4H), $7.16(\mathrm{~d}, 1 \mathrm{H}), 7.01-6.97(\mathrm{~m}, 2 \mathrm{H}), 6.85(\mathrm{~d}, 1 \mathrm{H}), 5.08(\mathrm{~s}, 2 \mathrm{H}), 3.66(\mathrm{~s}, 1 \mathrm{H}), 3.29(\mathrm{~s}, 4 \mathrm{H}), 3.10$ (s, $1 \mathrm{H}), 2.54(\mathrm{~s}, 6 \mathrm{H})$. Anal. Calcd for $\mathrm{C}_{37} \mathrm{H}_{36} \mathrm{FN}_{7} \mathrm{O}_{4} \mathrm{~S}(\%): \mathrm{C}, 64.05 ; \mathrm{H}, 5.23$; N, 14.13; Found (\%): C, $64.02 ; \mathrm{H}, 5.20 ; \mathrm{N}, 14.18$.

1-(4-(4-((4-(2-(2-(5-((4-Chlorobenzyl)oxy)-2-hydroxybenzylidene)hydrazinyl)-2-oxoethyl)piperazin-1-yl) methyl)thiazol-2-yl)phenyl)-3-(2-(trifluoromethyl)phenyl)urea (2i). Yield: 72.6\%; M.p.: 247-249 ${ }^{\circ} \mathrm{C}$; ESI-MS m/z: $778.0[\mathrm{M}-2 \mathrm{HCl}+\mathrm{H}]^{+}$; ${ }^{1} \mathrm{H}-\mathrm{NMR}$ (DMSO- $\left.d_{6}\right) \delta(\mathrm{ppm}): 12.03(\mathrm{~s}, 1 \mathrm{H}), 10.65(\mathrm{~s}, 1 \mathrm{H})$, $10.33-10.26(\mathrm{~m}, 3 \mathrm{H}), 8.50(\mathrm{~s}, 3 \mathrm{H}), 8.33(\mathrm{~s}, 1 \mathrm{H}), 7.86(\mathrm{~s}, 1 \mathrm{H}), 7.71(\mathrm{~s}, 2 \mathrm{H}), 7.47(\mathrm{~s}, 3 \mathrm{H}), 7.36-7.29$ $(\mathrm{m}, 4 \mathrm{H}), 6.98(\mathrm{dd}, 1 \mathrm{H}), 6.89(\mathrm{~d}, 1 \mathrm{H}), 5.06(\mathrm{~s}, 2 \mathrm{H}), 4.67(\mathrm{~s}, 2 \mathrm{H}), 4.52(\mathrm{~s}, 2 \mathrm{H}), 3.92-3.32(\mathrm{~s}, 8 \mathrm{H})$. Anal. Calcd for $\mathrm{C}_{38} \mathrm{H}_{35} \mathrm{ClF}_{3} \mathrm{~N}_{7} \mathrm{O}_{4} \mathrm{~S}(\%): \mathrm{C}, 58.65 ; \mathrm{H}, 4.53 ; \mathrm{N}, 12.60$; Found (\%): C, 58.63; H, 4.55; $\mathrm{N}, 12.61$. 
1-(4-Chloro-3-(Trifluoromethyl)phenyl)-3-(4-(4-((4-(2-(2-(5-((2-fluorobenzyl)oxy)-2-hydroxybenzylidene) hydrazinyl)-2-oxoethyl)piperazin-1-yl)methyl)thiazol-2-yl)phenyl)urea (2j). Yield: 74.5\%; M.p.: 165-167 ${ }^{\circ} \mathrm{C}$; ESI-MS m/z: 796.5 [M-2HCl+H] ${ }^{+} ;{ }^{1} \mathrm{H}-\mathrm{NMR}$ (DMSO- $\left.d_{6}\right) \delta(\mathrm{ppm}): 11.42(\mathrm{~s}, 1 \mathrm{H}), 10.71(\mathrm{~s}$, $1 \mathrm{H}), 9.75(\mathrm{~s}, 1 \mathrm{H}), 9.61(\mathrm{~s}, 1 \mathrm{H}), 9.47(\mathrm{~s}, 1 \mathrm{H}), 8.49(\mathrm{~s}, 1 \mathrm{H}), 8.13(\mathrm{~s}, 1 \mathrm{H}), 7.83(\mathrm{~d}, 2 \mathrm{H}), 7.66-7.52(\mathrm{~m}, 6 \mathrm{H})$, $7.40(\mathrm{~s}, 2 \mathrm{H}), 7.23-7.17(\mathrm{~m}, 3 \mathrm{H}), 6.99(\mathrm{~d}, 1 \mathrm{H}), 6.85(\mathrm{~d}, 1 \mathrm{H}), 5.08(\mathrm{~s}, 2 \mathrm{H}), 3.66(\mathrm{~s}, 1 \mathrm{H}), 3.64(\mathrm{~s}, 1 \mathrm{H})$, $3.49(\mathrm{~s}, 1 \mathrm{H}), 3.31(\mathrm{~s}, 1 \mathrm{H}), 3.11(\mathrm{~s}, 2 \mathrm{H}), 2.54(\mathrm{~s}, 6 \mathrm{H})$. Anal. Calcd for $\mathrm{C}_{38} \mathrm{H}_{34} \mathrm{ClF}_{4} \mathrm{~N}_{7} \mathrm{O}_{4} \mathrm{~S}(\%): \mathrm{C}, 57.32$; H, 4.30; N, 12.31; Found (\%): C, 57.33; H, 4.35; N, 12.25 .

1-(3-Chloro-4-fluorophenyl)-3-(4-(4-((4-(2-(2-(5-((3-chlorobenzyl)oxy)-2-hydroxybenzylidene)hydrazinyl)2-oxoethyl)piperazin-1-yl)methyl)thiazol-2-yl)phenyl)urea (2k). Yield: 73.3\%; M.p.: 194-195 ${ }^{\circ} \mathrm{C}$; ESI-MS $m / z: 762.2[\mathrm{M}-2 \mathrm{HCl}+\mathrm{H}]^{+} ;{ }^{1} \mathrm{H}-\mathrm{NMR}\left(\mathrm{DMSO}_{-} \mathrm{d}_{6}\right) \delta(\mathrm{ppm}): 11.42(\mathrm{~s}, 1 \mathrm{H}), 10.67(\mathrm{~s}, 1 \mathrm{H}), 9.75(\mathrm{~s}, 1 \mathrm{H})$, $9.09(\mathrm{~s}, 1 \mathrm{H}), 8.98(\mathrm{~s}, 1 \mathrm{H}), 8.47(\mathrm{~s}, 1 \mathrm{H}), 7.86-7.80(\mathrm{~m}, 4 \mathrm{H}), 7.58(\mathrm{~d}, 2 \mathrm{H}), 7.51(\mathrm{~s}, 1 \mathrm{H}), 7.41(\mathrm{~s}, 4 \mathrm{H})$, $7.34(\mathrm{~d}, 2 \mathrm{H}), 7.15(\mathrm{~d}, 1 \mathrm{H}), 7.00-6.96(\mathrm{~m}, 1 \mathrm{H}), 6.84(\mathrm{~d}, 1 \mathrm{H}), 5.07(\mathrm{~s}, 2 \mathrm{H}), 3.66(\mathrm{~s}, 2 \mathrm{H}), 3.32(\mathrm{~s}, 2 \mathrm{H})$, $3.10(\mathrm{~s}, 2 \mathrm{H}), 2.54(\mathrm{~s}, 6 \mathrm{H})$. Anal. Calcd for $\mathrm{C}_{37} \mathrm{H}_{34} \mathrm{Cl}_{2} \mathrm{FN}_{7} \mathrm{O}_{4} \mathrm{~S}(\%): \mathrm{C}, 58.27 ; \mathrm{H}, 4.49 ; \mathrm{N}, 12.86$; Found (\%): C, 58.25; H, 4.45; N, 12.92.

1-(4-(4-((4-(2-(2-)((7-Hydroxy-4-methyl-2-oxo-2H-chromen-6-yl)methylene)hydrazinyl)-2-oxoethyl) piperazin-1-yl)methyl)thiazol-2-yl)phenyl)-3-phenylurea (3a). Yield: 75.2\%; M.p.: 243-245 ${ }^{\circ} \mathrm{C}$; ESI-MS $m / z: 652.1[\mathrm{M}-2 \mathrm{HCl}+\mathrm{H}]^{+} ;{ }^{1} \mathrm{H}-\mathrm{NMR}\left(\mathrm{DMSO}-d_{6}\right) \delta(\mathrm{ppm}): 12.19(\mathrm{~s}, 1 \mathrm{H}), 9.46(\mathrm{~d}, 1 \mathrm{H}), 9.18(\mathrm{~d}, 1 \mathrm{H})$, $9.00(\mathrm{~s}, 1 \mathrm{H}), 8.61(\mathrm{~s}, 1 \mathrm{H}), 7.91(\mathrm{~d}, 3 \mathrm{H}), 7.71(\mathrm{~d}, 1 \mathrm{H}), 7.62(\mathrm{~d}, 2 \mathrm{H}), 7.00-6.95(\mathrm{~d}, 2 \mathrm{H}), 7.29(\mathrm{t}, 2 \mathrm{H})$, $7.00-6.95(\mathrm{~m}, 2 \mathrm{H}), 6.26(\mathrm{~s}, 1 \mathrm{H}), 4.47(\mathrm{~s}, 2 \mathrm{H}), 3.47(\mathrm{~d}, 10 \mathrm{H}), 2.41(\mathrm{~s}, 3 \mathrm{H})$. Anal. Calcd for $\mathrm{C}_{34} \mathrm{H}_{33} \mathrm{~N}_{7} \mathrm{O}_{5} \mathrm{~S}(\%): \mathrm{C}, 62.66 ; \mathrm{H}, 5.10 ; \mathrm{N}, 15.04$; Found (\%): C, 62.61; H, 5.13; N, 15.02.

1-(4-(4-((4-(2-(2-((7-Hydroxy-4-methyl-2-oxo-2H-chromen-6-yl)methylene)hydrazinyl)-2-oxoethyl) piperazin-1-yl)methyl)thiazol-2-yl)phenyl)-3-(3-(trifluoromethyl)phenyl)urea (3b). Yield: 71.7\%; M.p.: 242-243 ${ }^{\circ} \mathrm{C}$; ESI-MS m/z: $\left.720.1[\mathrm{M}-2 \mathrm{HCl}+\mathrm{H}]\right]^{+}$; ${ }^{1} \mathrm{H}-\mathrm{NMR}$ (DMSO- $\left.d_{6}\right) \delta(\mathrm{ppm}): 12.53(\mathrm{~s}, 1 \mathrm{H}), 12.16(\mathrm{~s}$, 1H), $10.99(\mathrm{~s}, 1 \mathrm{H}), 9.96(\mathrm{~s}, 1 \mathrm{H}), 9.87(\mathrm{~s}, 1 \mathrm{H}), 8.98(\mathrm{~s}, 1 \mathrm{H}), 8.61(\mathrm{~s}, 1 \mathrm{H}), 8.01(\mathrm{~s}, 1 \mathrm{H}), 7.93(\mathrm{~d}, 3 \mathrm{H})$, $7.72(\mathrm{t}, 1 \mathrm{H}), 7.68-7.57(\mathrm{~m}, 3 \mathrm{H}), 7.52(\mathrm{t}, 1 \mathrm{H}), 7.32(\mathrm{~d}, 1 \mathrm{H}), 6.98-6.95(\mathrm{~m}, 1 \mathrm{H}), 6.26(\mathrm{~s}, 1 \mathrm{H}), 4.52(\mathrm{~s}$, $2 \mathrm{H}), 3.80$ (s, 2H), 3.59 (brs, $8 \mathrm{H}), 2.41$ (s, 3H). Anal. Calcd for $\mathrm{C}_{35} \mathrm{H}_{32} \mathrm{~F}_{3} \mathrm{~N}_{7} \mathrm{O}_{5} \mathrm{~S}(\%): \mathrm{C}, 58.41 ; \mathrm{H}, 4.48$; N, 13.62; Found (\%): C, 58.43; H, 4.43; N, 13.68 .

1-(4-(4-((4-(2-(2-((7-Hydroxy-4-methyl-2-oxo-2H-chromen-6-yl)methylene)hydrazinyl)-2-oxoethyl) piperazin-1-yl)methyl)thiazol-2-yl)phenyl)-3-(3-methoxyphenyl)urea (3c). Yield: 75.3\%; M.p.: 236-238 ${ }^{\circ} \mathrm{C}$; ESI-MS $m / z$ : $682.3[\mathrm{M}-2 \mathrm{HCl}+\mathrm{H}]^{+} ;{ }^{1} \mathrm{H}-\mathrm{NMR}\left(\mathrm{DMSO}-d_{6}\right) \delta(\mathrm{ppm}): 12.60(\mathrm{~s}, 1 \mathrm{H}), 12.19(\mathrm{~s}, 1 \mathrm{H}), 9.40(\mathrm{~s}$, 1H), $9.00(\mathrm{~s}, 2 \mathrm{H}), 8.61(\mathrm{~s}, 1 \mathrm{H}), 7.89(\mathrm{~s}, 3 \mathrm{H}), 7.74(\mathrm{~d}, 1 \mathrm{H}), 7.61(\mathrm{~d}, 2 \mathrm{H}), 7.37(\mathrm{~d}, 2 \mathrm{H}), 6.97(\mathrm{~d}, 1 \mathrm{H}), 6.88$ $(\mathrm{d}, 2 \mathrm{H}), 6.26(\mathrm{~s}, 1 \mathrm{H}), 4.47(\mathrm{~s}, 2 \mathrm{H}), 3.72(\mathrm{~s}, 3 \mathrm{H}), 3.61(\mathrm{~s}, 10 \mathrm{H}), 2.41(\mathrm{~s}, 3 \mathrm{H})$. Anal. Calcd for $\mathrm{C}_{35} \mathrm{H}_{35} \mathrm{~N}_{7} \mathrm{O}_{6} \mathrm{~S}(\%): \mathrm{C}, 61.66 ; \mathrm{H}, 5.17 ; \mathrm{N}, 14.38$; Found (\%): C, 61.13; H, 5.14; N, 14.35.

1-(3,5-bis(Trifluoromethyl)phenyl)-3-(4-(4-((4-(2-(2-((7-hydroxy-4-methyl-2-oxo-2H-chromen-6-yl) methylene)hydrazinyl)-2-oxoethyl)piperazin-1-yl)methyl)thiazol-2-yl)phenyl)urea (3d). Yield: 70.1\%; M.p.: $225-226{ }^{\circ} \mathrm{C}$; ESI-MS m/z: $788.3[\mathrm{M}-2 \mathrm{HCl}+\mathrm{H}]^{+} ;{ }^{1} \mathrm{H}-\mathrm{NMR}\left(\mathrm{DMSO}-d_{6}\right) \delta$ (ppm): $12.68(\mathrm{~s}, 1 \mathrm{H})$, $10.53(\mathrm{~d}, 1 \mathrm{H}), 10.01(\mathrm{~d}, 1 \mathrm{H}), 8.98(\mathrm{~s}, 1 \mathrm{H}), 8.13(\mathrm{~s}, 2 \mathrm{H}), 8.00-7.89(\mathrm{~m}, 4 \mathrm{H}), 7.73(\mathrm{t}, 1 \mathrm{H}), 7.66(\mathrm{~d}, 3 \mathrm{H})$, 
7.06-6.88 (m, 1H), $6.26(\mathrm{~d}, 1 \mathrm{H}), 4.55(\mathrm{~s}, 4 \mathrm{H}), 4.11-3.56(\mathrm{~m}, 8 \mathrm{H}), 2.41(\mathrm{~s}, 3 \mathrm{H})$. Anal. Calcd for $\mathrm{C}_{36} \mathrm{H}_{31} \mathrm{~F}_{6} \mathrm{~N}_{7} \mathrm{O}_{5} \mathrm{~S}(\%): \mathrm{C}, 54.89 ; \mathrm{H}, 3.97 ; \mathrm{N}, 12.45$; Found (\%): C, 54.88; H, 3.94; N, 12.40.

1-(3,4-Dimethylphenyl)-3-(4-(4-((4-(2-(2-((7-hydroxy-4-methyl-2-oxo-2H-chromen-6-yl)methylene) hydrazinyl)-2-oxoethyl)piperazin-1-yl)methyl)thiazol-2-yl)phenyl)urea (3e). Yield: 72.2\%; M.p.: 246-248 ${ }^{\circ} \mathrm{C}$; ESI-MS m/z: $666.3[\mathrm{M}-2 \mathrm{HCl}+\mathrm{H}]^{+}$; ${ }^{1} \mathrm{H}-\mathrm{NMR}$ (DMSO- $\left.d_{6}\right) \delta(\mathrm{ppm}): 12.56(\mathrm{~s}, 1 \mathrm{H}), 12.16$ $(\mathrm{s}, 1 \mathrm{H}), 11.00(\mathrm{~s}, 1 \mathrm{H}), 9.71(\mathrm{~d}, 1 \mathrm{H}), 9.26(\mathrm{~d}, 1 \mathrm{H}), 8.98(\mathrm{~s}, 1 \mathrm{H}), 7.93-7.88(\mathrm{~m}, 3 \mathrm{H}), 7.75-7.70(\mathrm{~m}, 1 \mathrm{H})$, $7.61(\mathrm{dd}, 2 \mathrm{H}), 7.24-7.19(\mathrm{~m}, 2 \mathrm{H}), 7.04-6.95(\mathrm{~m}, 2 \mathrm{H}), 6.27-6.25(\mathrm{~m}, 1 \mathrm{H}), 4.52(\mathrm{~s}, 2 \mathrm{H}), 3.81(\mathrm{~s}, 2 \mathrm{H})$, 3.60 (brs, 8H), 2.41 (s, 3H), 2.19 (s, 3H), 2.16 (s, 3H). Anal. Calcd for $\mathrm{C}_{36} \mathrm{H}_{37} \mathrm{~N}_{7} \mathrm{O}_{5} \mathrm{~S}(\%): \mathrm{C}, 63.61 ; \mathrm{H}$, $5.49 ; \mathrm{N}, 14.42$; Found (\%): C, 63.58; H, 5.54; N, 14.40.

\subsection{General Procedure for Preparation of Target Compounds 4a-c}

Acethydrazide 11e or $110(0.002 \mathrm{~mol})$ was dissolved in $1 \mathrm{M}$ hydrochloride $(5 \mathrm{~mL})$ to obtain the hydrochloride form. The resulting hydrochloride salt was then added to a solution of imidazolindione 19a-b $(0.002 \mathrm{~mol})$ in ethanol $(10 \mathrm{~mL})$. The reaction mixture was stirred at room temperature for $4 \mathrm{~h}$. The precipitates were filtered off and dried to obtain $\mathbf{4 a - c}$ as dihydrochlorides.

1-(3-Chloro-4-(trifluoromethyl)phenyl)-3-(4-(4-((4-(2-(2-((2,5-dioxo-1-(4-(trifluoromethyl)phenyl) imidazolidin-4-yl)methylene)hydrazinyl)-2-oxoethyl)piperazin-1-yl)methyl)thiazol-2-yl)phenyl)urea (4a). Yield: 58.4\%; M.p.: 239-241 ${ }^{\circ} \mathrm{C}$; ESI-MS m/z: $822.0[\mathrm{M}-2 \mathrm{HCl}+\mathrm{H}]^{+} ;{ }^{1} \mathrm{H}-\mathrm{NMR}\left(\mathrm{DMSO}-d_{6}\right) \delta$ (ppm): $10.63(\mathrm{~s}, 1 \mathrm{H}), 9.98(\mathrm{~s}, 2 \mathrm{H}), 9.77(\mathrm{~s}, 1 \mathrm{H}), 8.72(\mathrm{~d}, 1 \mathrm{H}), 8.12(\mathrm{~s}, 1 \mathrm{H}), 7.94(\mathrm{~s}, 1 \mathrm{H}), 7.92(\mathrm{~d}$, $J=3.1 \mathrm{~Hz}, 2 \mathrm{H}), 7.84(\mathrm{~d}, 2 \mathrm{H}), 7.67(\mathrm{~d}, 2 \mathrm{H}), 7.65-7.62(\mathrm{t}, 4 \mathrm{H}), 6.73(\mathrm{~d}, 1 \mathrm{H}), 4.49(\mathrm{~s}, 2 \mathrm{H}), 3.73(\mathrm{~s}, 2 \mathrm{H})$, 3.38 (brs, 8H). Anal. Calcd for $\mathrm{C}_{35} \mathrm{H}_{30} \mathrm{ClF}_{6} \mathrm{~N}_{9} \mathrm{O}_{4} \mathrm{~S}(\%): \mathrm{C}, 51.13 ; \mathrm{H}, 3.68 ; \mathrm{N}, 15.33$; Found (\%): C, 51.18; H, 3.64; N, 15.40 .

1-(4-(4-((4-(2-(2-((2,5-dioxo-1-(4-(Trifluoromethyl)phenyl)imidazolidin-4-yl)methylene)hydrazinyl)-2oxoethyl)piperazin-1-yl)methyl)thiazol-2-yl)phenyl)-3-(3-(trifluoromethyl)phenyl)urea (4b). Yield: 57.8\%; M.p.: $205-207{ }^{\circ} \mathrm{C}$; ESI-MS m/z: $788.0[\mathrm{M}-2 \mathrm{HCl}+\mathrm{H}]^{+} ;{ }^{1} \mathrm{H}-\mathrm{NMR}$ (DMSO-d $d_{6} \delta(\mathrm{ppm}): 10.32(\mathrm{~s}, 1 \mathrm{H})$, $9.61(\mathrm{~d}, 3 \mathrm{H}), 8.02(\mathrm{~s}, 1 \mathrm{H}), 7.89-7.83(\mathrm{~m}, 5 \mathrm{H}), 7.70-7.50(\mathrm{~m}, 9 \mathrm{H}), 7.32(\mathrm{~d}, 1 \mathrm{H}), 4.43(\mathrm{~s}, 2 \mathrm{H}), 3.32(\mathrm{~s}$, 10H). Anal. Calcd for $\mathrm{C}_{35} \mathrm{H}_{31} \mathrm{~F}_{6} \mathrm{~N}_{9} \mathrm{O}_{4} \mathrm{~S}(\%): \mathrm{C}, 53.37$; H, 3.97; N, 16.00; Found (\%): C, 53.38; H, 3.94; $\mathrm{N}, 15.92$.

1-(4-(4-((4-(2-(2-((1-(4-Fluorophenyl)-2,5-dioxoimidazolidin-4-yl)methylene)hydrazinyl)-2-oxoethyl) piperazin-1-yl)methyl)thiazol-2-yl)phenyl)-3-(3-(trifluoromethyl)phenyl)urea (4c). Yield: 55.2\%; M.p.: 200-201 ${ }^{\circ} \mathrm{C}$; ESI-MS $\left.m / z: 738.2[\mathrm{M}-2 \mathrm{HCl}+\mathrm{H}]\right]^{+}$; ${ }^{1} \mathrm{H}-\mathrm{NMR}$ (DMSO- $\left.d_{6}\right) \delta(\mathrm{ppm}): 11.56(\mathrm{~s}, 1 \mathrm{H}), 10.22(\mathrm{~s}$, $1 \mathrm{H}), 9.60-9.35(\mathrm{~m}, 3 \mathrm{H}), 8.31(\mathrm{~d}, 1 \mathrm{H}), 8.03(\mathrm{~s}, 1 \mathrm{H}), 7.90(\mathrm{~d}, 2 \mathrm{H}), 7.65-7.60(\mathrm{~m}, 3 \mathrm{H}), 7.55-7.50(\mathrm{~m}$, 1H), 7.45-7.26 (m, 5H), $6.65(\mathrm{~d}, 1 \mathrm{H}), 4.23(\mathrm{~s}, 2 \mathrm{H}), 3.75(\mathrm{~s}, 1 \mathrm{H}), 3.52-2.55(\mathrm{~m}, 9 \mathrm{H})$. Anal. Calcd for $\mathrm{C}_{34} \mathrm{H}_{31} \mathrm{~F}_{4} \mathrm{~N}_{9} \mathrm{O}_{4} \mathrm{~S}(\%): \mathrm{C}, 55.35 ; \mathrm{H}, 4.24 ; \mathrm{N}, 17.09$; Found (\%): C, 55.38; H, 4.20; N, 17.12.

\subsection{Evaluation of the Biological Activity}

The cytotoxicity of target compounds $1 \mathbf{a}-\mathbf{g}, \mathbf{2 a}-\mathbf{k}, \mathbf{3 a}-\mathbf{e}$ and $\mathbf{4 a}-\mathbf{c}$ were evaluated against the A549, MDA-MB-231 and HL-60 cell lines by MTT method in vitro, with sorafenib and PAC-1 as the 
positive controls. The cancer cell lines were cultured in minimum essential medium (MEM) supplement with $10 \%$ fetal bovine serum (FBS). Approximately $4 \times 10^{3}$ cells, suspend in MEM medium, were plated onto each well of a 96-well plate and incubated in $5 \% \mathrm{CO}_{2}$ at $37{ }^{\circ} \mathrm{C}$ for $24 \mathrm{~h}$. The tested compounds at indicated final concentrations were added to the culture medium and the cell cultures were continued for $72 \mathrm{~h}$. Fresh MTT was added to each well at a terminal concentration of $5 \mu \mathrm{g} / \mathrm{mL}$ and incubated with cells at $37{ }^{\circ} \mathrm{C}$ for $4 \mathrm{~h}$. The formazan crystals were dissolved in $100 \mu \mathrm{L}$ DMSO per well, and the absorbency at $492 \mathrm{~nm}$ (for absorbance of MTT formazan) and $630 \mathrm{~nm}$ (for the reference wavelength) was measured with the ELISA reader. All of the compounds were tested twice in the cell lines. The results expressed as $\mathrm{IC}_{50}$ (inhibitory concentration of 50\%) were the averages of two determinations and were calculated by using the Bacus Laboratories Incorporated Slide Scanner (Bliss) software.

\section{Conclusions}

In summary, a novel series of dual diaryl urea and $N$-acylhydrazone derivatives were designed based on the hybrid pharmacophore concept. All the target compounds were synthesized and screened for their cytotoxicity against three human cancer cell lines (HL-60, A549 and MDA-MB-231) by standard MTT assays. The pharmacological results indicated that most compounds exhibited moderate to excellent activity. The preliminary SARs showed that both electron-withdrawing groups on $\operatorname{Ar}^{1}$ and 4- or 5-benzyloxyl groups on $\mathrm{Ar}^{2}$ are favorable for optimal cytotoxicity. Moreover, this encouraging research provides a valuable leading compound $2 \mathrm{~g}$ with $\mathrm{IC}_{50}$ values of $0.22,0.34$ and $0.41 \mu \mathrm{M}$ against tested cell lines respectively, which were 3.8 to 22.5 times more active than the references sorafenib and PAC-1, and highlights the potential for further development of novel dual diaryl urea and $\mathrm{N}$-acylhydrazone derivatives. Studies on the mechanism of action of these compounds are in progress and will be reported in the near future.

\section{Acknowledgements}

This work was supported by the National Natural Science Foundation of China (No. 21002065) and the Young Scholar Growth Plan in University of Liaoning Province (No. LJQ201107).

\section{References}

1. Keating, G.M.; Santoro, A. Sorafenib: A review of its use in advanced hepatocellular carcinoma. Drugs 2009, 69, 223-240.

2. Wilhelm, S.M.; Adnane, L.; Newell, P.; Villanueva, A.; Llovet, J.M.; Lynch, M. Preclinical overview of sorafenib, a multikinase inhibitor that targets both Raf and VEGF and PDGF receptor tyrosine kinase signaling. Mol. Cancer Ther. 2008, 7, 3129-3140.

3. Smalley, K.S.; Xiao, M.; Villanueva, J.; Nguyen, T.K.; Flaherty, K.T.; Letrero, R.; van Belle, P.; Elder, D.E.; Wang, Y.; Nathanson, K.L.; et al. CRAF inhibition induces apoptosis in melanoma cells with non-V600E BRAF mutations. Oncogene 2009, 28, 85-94. 
4. Adnane, L.; Trail, P.A.; Taylor, I.; Wilhelm, S.M. Sorafenib (BAY 43-9006, Nexavar), a dual-action inhibitor that targets RAF/MEK/ERK pathway in tumor cells and tyrosine kinases VEGFR/PDGFR in tumor vasculature. Methods Enzymol. 2006, 407, 597-612.

5. Hyun, Y.W.; Jeong, H. Sorafenib in liver cancer. Expert Opin. Pharmacother. 2012, 13, 1059-1067.

6. Strumberg, D. Sorafenib for the treatment of renal cancer. Expert Opin. Pharmacother. 2012, 13, 407-419.

7. Peterson, Q.P.; Hsu, D.C.; Goode, D.R.; Novotny, C.J.; Totten, R.K.; Hergenrother, P.J. Procaspase-3 activation as an anti-cancer strategy: Structure-activity relationship of PAC-1, and its cellular co-localization with caspase-3. J. Med. Chem. 2009, 52, 5721-5731.

8. Lucas, P.W.; Schmit, J.M.; Peterson, Q.P.; West, D.C.; Hsu, D.C.; Novotny, C.J.; Dirikolu, L.; Churchwell, M.I.; Doerge, D.R.; Garrett, L.D.; et al. Pharmacokinetics and Derivation of an anticancer dosing regimen for PAC-1, a preferential small molecule activator of procaspase-3, in healthy dogs. Invest. New Drugs 2011, 29, 901-911.

9. Peterson, Q.P.; Goode, D.R.; West, D.C.; Ramsey, K.N.; Lee, J.J.; Hergenrother, P.J. PAC-1 activates procaspase-3 in vitro through relief of zinc-mediated inhibition. J. Mol. Biol. 2009, 388, $144-158$.

10. Zhang, B.; Zhao, Y.; Zhai, X.; Wang, L.; Yang, J.; Tan, Z.; Gong, P. Design, synthesis and anticancer activities of diaryl urea derivatives bearing $\mathrm{N}$-acylhydrazone moiety. Chem. Pharm. Bull. 2012, 60, 1046-1054.

11. Putt, K.S.; Chen, G.W.; Pearson, J.M.; Sandhorst, J.S.; Hoagland,M.S.; Kwon, J.T.; Hwang, S.K.; Jin, H.; Churchwell, M.I.; Cho, M.H.; et al. Small-molecule activation of procaspase-3 to caspase-3 as a personalized anticancer strategy. Nat. Chem. Biol. 2006, 2, 543-550.

12. Kudo, M.; Hanashima, T.; Muranaka, A.; Sato, H.; Uchiyama, M.; Azumaya, I.; Hirano, T.; Kagechika, H.; Tanatani, A. Identification of Absolute Helical Structures of Aromatic Multilayered Oligo(m-Phenylurea)s in Solution. J. Org. Chem. 2009, 74, 8154-8163.

13. Duff, J.C.; Bills, E.J. Reactions between Hexamethylenetetramine and Phenolic Compounds. Part I. A New Method for the Preparation of 3- and 5-Aldehydosalicylic Acids. J. Chem. Soc. 1932, 1932, 1987-1988.

14. Muccioli, G.G.; Fazio, N.; Scriba, G.K.; Poppitz, W.; Cannata, F.; Poupaert, J.H.; Wouters, J.; Lambert, J.D. Substituted 2-Thioxoimidazolidin-4-Ones and Imidazolidine-2,4-Diones as Fatty Acid Amide Hydrolase Inhibitors Templates. J. Med. Chem. 2006, 49, 417-425.

Sample Availability: Samples of the compounds are available from the authors.

(C) 2013 by the authors; licensee MDPI, Basel, Switzerland. This article is an open access article distributed under the terms and conditions of the Creative Commons Attribution license (http://creativecommons.org/licenses/by/3.0/). 